\title{
Constraints on the resistivity of the oceanic lithosphere and asthenosphere from seafloor ocean tidal electromagnetic measurements
}

\author{
H. Zhang, ${ }^{1,2}$ G.D. Egbert, ${ }^{2}$ A.D. Chave ${ }^{\oplus},{ }^{3}$ Q. Huang, ${ }^{1}$ A. Kelbert ${ }^{4}$ and S.Y. Erofeeva ${ }^{2}$ \\ ${ }^{1}$ School of Earth and Space Sciences, Peking University, Beijing, China.E-mail: huangq@pku.edu.cn \\ ${ }^{2}$ College of Earth, Ocean, and Atmospheric Sciences, Oregon State University, OR, USA \\ ${ }^{3}$ Department of Applied Ocean Physics and Engineering, Woods Hole Oceanographic Institution, MA, USA \\ ${ }^{4}$ USGS Geomagnetism Program, Golden, CO, USA
}

Accepted 2019 July 10. Received 2019 March 15; in original form 2019 July 1

\section{SUMMAR Y}

The electromagnetic (EM) field generated by ocean tidal flow is readily detectable in both satellite magnetic field data, and in ocean-bottom measurements of electric and magnetic fields. The availability of accurate charts of tidal currents, constrained by assimilation of modern satellite altimetry data, opens the possibility of using tidal EM fields as a source to image mantle electrical resistivity beneath the ocean basins, as highlighted by the recent success in defining the globally averaged lithosphere-asthenosphere boundary (LAB) with satellite data. In fact, seafloor EM data would be expected to provide better constraints on the structure of resistive oceanic lithosphere, since the toroidal magnetic mode, which can constrain resistive features, is a significant component of the tidal EM field within the ocean, but is absent above the surface (in particular in satellite data). Here we consider this issue in more detail, using a combination of simplified theoretical analysis and 1-D and 3-D numerical modelling to provide a thorough discussion of the sensitivity of satellite and seafloor data to subsurface electrical structure. As part of this effort, and as a step toward 3-D inversion of seafloor tidal data, we have developed a new flexible 3-D spherical-coordinate finite difference scheme for both global and regional scale modelling, with higher resolution models nested in larger scale solutions. We use the new 3-D model, together with Monte Carlo simulations of errors in tidal current estimates, to provide a quantitative assessment of errors in the computed tidal EM signal caused by uncertainty in the tidal source. Over the open ocean this component of error is below $0.01 \mathrm{nT}$ in $B_{z}$ at satellite height and $0.05 \mathrm{nT}$ in $B_{x}$ on the seafloor, well below typical signal levels. However, as coastlines are approached error levels can increase substantially. Both analytical and 3-D modelling demonstrate that the seafloor magnetic field is most sensitive to the lithospheric resistance (the product of resistivity and thickness), and is more weakly influenced (primarily in the phase) by resistivity of the underlying asthenosphere. Satellite data, which contain only the poloidal magnetic mode, are more sensitive to the conductive asthenosphere, but have little sensitivity to lithospheric resistance. For both seafloor and satellite data's changes due to plausible variations in Earth parameters are well above error levels associated with source uncertainty, at least in the ocean interior. Although the 3-D modelling results are qualitatively consistent with theoretical analysis, the presence of coastlines and bathymetric variations generates a complex response, confirming that quantitative interpretation of ocean tidal EM fields will require a 3-D treatment. As an illustration of the nested 3-D scheme, seafloor data at five magnetic and seven electric stations in the northeastern Pacific $\left(41^{\circ} \mathrm{N}, 165^{\circ} \mathrm{W}\right)$ are fit with trial-and-error forward modelling of a local domain. The simulation results indicate that the lithospheric resistance is roughly $7 \times 10^{8} \Omega \mathrm{m}^{2}$. The phase of the seafloor data in this region are inconsistent with a sharp transition between the resistive lithosphere and conductive asthenosphere.

Key words: Composition and structure of the mantle; Pacific Ocean; Electromagnetic theory; Geomagnetic induction; Satellite magnetics. 


\section{INTRODUCTION}

Electrical resistivity can provide important constraints on the physical state and evolution of Earth. While the electrical structure of the lithosphere and asthenosphere remain relatively poorly explored beneath the ocean basins compared to the continents, the last few decades have seen substantial progress in understanding the resistivity of the oceanic lithosphere and asthenosphere. On one hand, numerous long period seafloor magnetotelluric (MT) experiments have been conducted to investigate regional deep structure (e.g. Filloux 1977; Wannamaker et al. 1989; Evans et al. 1999; Baba et al. 2006; Matsuno et al. 2010; Key et al. 2013; Naif et al. 2013). On the other hand, resistivity models from satellite magnetic measurements (Kuvshinov \& Olsen 2006; Velímskỳ et al. 2006; Püthe et al. 2015; Grayver et al. 2017) and geomagnetic observatory data (Kelbert et al. 2009; Munch et al. 2018) have shed light on the global electrical structure. In ocean basins far from active tectonic regions like mid-ocean ridges and subduction zones, the typical electrical resistivity structure is basically $1-\mathrm{D}$, consisting of a resistive oceanic lithosphere and an underlying conductive asthenosphere (Sarafian et al. 2015), although the study of the northwestern Pacific (Baba et al. 2017) does suggest that both the lithosphere and asthenosphere exhibit lateral variations on a large scale.

Recently, the use of ocean tidal EM (OTEM) fields to image electrical resistivity beneath the ocean basins has drawn significant attention. Generated by the gravitationally-forced tide that drives conductive seawater through Earth's ambient magnetic field, the OTEM signal has been detected in ground (Junge 1988; Maus \& Kuvshinov 2004; Schnepf et al. 2018), seafloor (Larsen 1968; Luther et al. 1991; Kuvshinov et al. 2006; Schnepf et al. 2014) and satellite (Tyler et al. 2003; Sabaka et al. 2015) measurements. The OTEM approach has two potential advantages over other natural source induction methods, such as MT and Geomagnetic Deep Sounding (GDS). First, both the poloidal magnetic (PM) and toroidal magnetic (TM) modes are produced in a 1-D Earth (Chave \& Luther 1990) by the OTEM source. Chave (1984) examined the Fréchet derivatives of the PM and TM modes in EM induction and confirmed that the TM mode possesses superior resolution for resistive structure. Compared with purely induction methods that exclusively rely on the PM mode, the TM mode of the OTEM field is much less biased towards low resistivity anomalies, making it more suitable to constrain the resistivity of the oceanic lithosphere. Second, current global models of ocean tidal flows are of high accuracy, with the source frequency-wavenumber structure much better known for the OTEM source than for the ionospheric and magnetospheric sources used for MT and GDS.

Early geophysical studies on motional induction relied on simplified analytical models of ocean movement (Larsen 1968), and adopted analytical approaches to elucidate basic physics (e.g. Sanford 1971; Chave 1983). These studies demonstrated the potential value of using OTEM to probe Earth's resistivity, but available source models were not realistic enough for quantitative applications. Moreover, 1-D analytical methods failed to incorporate the coast effect which originates from the sharp resistivity contrast between the seawater and continent, and has been generally recognized in GDS and MT (e.g. Parkinson \& Jones 1979; Key \& Constable 2011). The modern era of OTEM studies essentially began with the development of high-resolution global ocean tide models from the TOPEX/Poseidon and subsequent radar altimetry missions (e.g. Le Provost 2001; Stammer et al. 2014; Ray \& Egbert 2017). For the first time, the altimeter provided accurate measurements of tidal seasurface height in the open ocean. Through assimilation of these data into a hydrodynamic model, estimates of open ocean barotropic (essentially depth independent) tidal currents are now available across the globe (Egbert et al. 1994; Egbert \& Erofeeva 2002; Taguchi et al. 2014). Stammer et al. (2014) compared various models (including purely hydrodynamic models) with validation data, and concluded that modern models of tidal currents are reliable, at least in the open ocean.

More recent studies on OTEM have been based on satellite data from the CHAMP and Swarm magnetic field missions. After it was shown that the M2 tide could be extracted from CHAMP data (Tyler et al. 2003), attention has turned to use of these data to make inferences about Earth's resistivity (Schnepf et al. 2015; Grayver et al. 2016; Grayver \& Olsen 2019) and global ocean heat content (Irrgang et al. 2019). Generally speaking, the main focus of these studies has been on the radial component of the satellite magnetic field, and a numerical modelling approach based on the integral equation method is implemented for a heterogeneous surface shell with an underlying 1-D global resistivity model (Maus \& Kuvshinov 2004). More comprehensive descriptions can be found in the review papers of Kuvshinov (2008) and Minami (2017).

However, the toroidal magnetic field, associated with electric currents with a vertical component, vanishes at an insulating surface. The TM part of the OTEM signal does not penetrate the atmosphere, hence is absent in satellite data and only measurable inside of Earth (e.g. horizontal magnetic fields on the seafloor). Ocean-bottom EM observations collected in numerous campaigns in the Pacific over the past $30+$ years provide a potentially valuable database of this TM signal. Here we emphasize seafloor horizontal magnetic fields, and explore how these data can provide additional constraints on the oceanic lithosphere and asthenosphere. We focus on the lunar semidiurnal (M2) tide, the strongest constituent in the tidal spectrum with a period of $\sim 12.42 \mathrm{hr}$.

Considering the future goal of simultaneous inversion of multiple seafloor EM datasets, we have developed a new modelling tool for the OTEM field that can easily handle a 3-D arbitrary electrical resistivity distribution, which is required for accurate prediction in coastal areas (Velímský et al. 2018). The algorithm utilizes a nested spherical-coordinates finite difference (FD) scheme, with higher resolution regional models embedded in lower resolution (ultimately global) models. Based on theoretical study of low-frequency, motionally induced EM fields (Chave \& Luther 1990), we explore the relation between tidal flow and the TM and PM modes of the OTEM field. Although the tidal velocity fields that drive electric source currents are realistic, they are not perfect. We use the new modelling tool to assesses how errors in tidal velocity estimates propagate to the modelled OTEM fields, using the Monte Carlo error propagation approach described in Egbert et al. (1994) and Ray et al. (2001). We also combine the semi-quantitative analysis and numerical simulations to gauge the sensitivity of surface/satellite and seafloor OTEM data to lithospheric and asthenospheric resistivity.

Finally, OTEM signals at five magnetic and seven electric stations from the northeastern Pacific Ocean bottom BEMPEX experiment (Luther et al. 1987) are fitted by using trial-and-error high resolution regional forward modelling. Schnepf et al. (2014) processed and modelled the seafloor OTEM signal (amplitude of total field) from six magnetic stations in the northwestern Pacific Ocean, but there exist discrepancies between their observations and predictions, using the regional 1-D resistivity models revealed by MT data at the same sites (Baba et al. 2010). Comparisons between the best-fitting synthetic resistivity model and models from previous marine MT studies demonstrates that seafloor OTEM data can put new constraints on the electrical properties of Earth's deep interior. 
The paper is organized as follows. The basic theory of OTEM modes and semi-quantitative analysis of sensitivity are given in Section 2. A new OTEM forward modelling tool based on a 3-D FD method in spherical coordinates that enables modelling at global and regional scales is described in Section 3. Section 4 presents estimates of error propagated to the modelled OTEM fields from the uncertainty in the tidal model. Section 5 further discusses the sensitivity of PM and TM modes by using numerical modelling results. Section 6 compares the seafloor observations in the BEMPEX experiment with predictions from high-resolution local forward modelling.

\section{THEORY}

Motional induction by ocean tides is described by the Maxwell equations under the magneto-quasistatic approximation in the frequency domain. Assuming harmonic time dependence $e^{-i \omega t}$, the electric field $\mathbf{E}$ satisfies the following second order partial differential equation:

$\nabla \times \nabla \times \mathbf{E}-i \omega \mu \sigma \mathbf{E}=i \omega \mu \mathbf{J}_{\mathbf{s}}$

$\mathbf{J}_{\mathbf{s}}=\sigma_{s}\left(\mathbf{v} \times \mathbf{B}^{\mathbf{m}}\right)$.

The right-hand term $\mathbf{J}_{\mathbf{s}}$ is the electric source current caused by ocean tides. $\mathbf{v}$ is tidal flow velocity, $\sigma_{s}$ is the seawater electrical conductivity and $\mathbf{B}^{\mathbf{m}}$ is the main geomagnetic field that is assumed to be time-independent. Fig. 1 illustrates the tidal current (in phase and quadrature components) of the M2 constituent in the tidal model TPXO8 (Egbert \& Erofeeva 2002). Corresponding surface height variations and cotidal lines are shown in Fig. S2.

\subsection{PM and TM modes}

The modal form can reduce a vector field into simpler parts with distinct physics. To theoretically explore how the tidal flow type and deep electrical structure influence OTEM, the analytical approach of Backus (1986) and Chave \& Luther (1990) was used to decompose the magnetic field into TM and PM modes. We clarify here that this section aims to catch sight of the basic features of the OTEM field under certain simplifying assumptions, to help provide a qualitative explanation for numerical modelling results in the following sections. The explicit expression of modal potential functions under rigorous conditions is out of this paper's scope.

The magnetic field $\mathbf{B}$ can be represented by poloidal and toroidal scalar functions $\Psi$ and $\Pi$ in spherical coordinate:

$\mathbf{B}=\hat{\mathbf{r}} \times \nabla_{h} \Pi+\nabla_{h} \partial_{r} \Psi-\nabla_{h}^{2} \Psi \hat{\mathbf{r}}$,

where $\hat{\mathbf{r}}$ is the radial unit vector, and $\nabla_{h}=\nabla-\hat{\mathbf{r}} \partial_{r}$ is the surface gradient on a sphere. The PM mode scalar $\Psi$ is produced by horizontal electric current loops that couple through induction, and has no vertical electric field component. The TM mode scalar $\Pi$ is produced by electric current loops with vertical component, and has no vertical magnetic field component. In a homogeneous 1-D earth, satellite data and the seafloor/land vertical magnetic field only contain the PM mode, while the seafloor horizontal magnetic fields contain both modes.

Following Chave \& Luther (1990), the electric source current term $\mathbf{J}_{\mathbf{s}}$ can be expanded into consoidal, toroidal and radial components:

$\mathbf{J}_{\mathbf{s}}=\hat{\mathbf{r}} \times \nabla_{h} Y+\nabla_{h} T+\Xi \hat{\mathbf{r}}$.
In a layered earth with constant magnetic permeability, the PM scalar functions $\Psi$ is associated with $Y$, and the TM function $\Pi$ is associated with $T$ and $\Xi$. For long surface gravity waves like the ocean tide, the current velocity $\mathbf{v}$ is tangential to the surface and approximately uniform through the water column. Ignoring variations of $\mathbf{B}^{\mathbf{m}}$ and $\sigma_{s}$ through the ocean, replacing velocity $\mathbf{v}$ by the volume transport vector $\mathbf{V}$ (the vertical integral of $\mathbf{v}$ over the depth) in (2), we obtain an expression for the vertically integrated electric current density. As this effectively characterizes the OTEM source, and is the relevant input for thin sheet modelling (Maus \& Kuvshinov 2004; Kuvshinov et al. 2006), we consider the volume transport vector in the following.

According to the Helmholtz-Hodge decomposition, $\mathbf{V}$ can be written in terms of a potential $\phi$ and a stream function $\psi$ :

$\mathbf{V}=\nabla_{h} \phi+\hat{\mathbf{r}} \times \nabla_{h} \psi$.

The geomagnetic ambient field $\mathbf{B}^{\mathbf{m}}$ consists of the radial component $B_{r}^{m}$ and the horizontal component $\mathbf{B}_{\mathbf{h}}^{\mathbf{m}}$ :

$\mathbf{B}^{\mathbf{m}}=B_{r}^{m} \hat{\mathbf{r}}+\mathbf{B}_{\mathbf{h}}^{\mathbf{m}}$.

Substitute eqs (5) and (6) into (2). Even though there is substantial north-south gradient in both $\mathbf{B}^{\mathbf{m}}$ and $\sigma_{s}$, the characterized wavenumber of the tides is still smaller than planetary. Hence, to first order we can ignore spatial variations of $\mathbf{B}^{\mathbf{m}}$ and $\sigma_{s}$. Then, moving $\mathbf{B}^{\mathbf{m}}$ and $\sigma_{s}$ inside the differential operator, the electric source current can be approximated as:

$$
\begin{aligned}
\mathbf{J}_{\mathbf{s}}= & \hat{\mathbf{r}} \times \nabla_{h}\left(\sigma_{s} B_{r}^{m} \phi\right)+\nabla_{h}\left(\sigma_{s} B_{r}^{m} \psi\right) \\
& +\sigma_{s}\left(\nabla_{h} \phi \times \mathbf{B}_{\mathbf{h}}^{\mathbf{m}}-\nabla_{h} \psi \cdot \mathbf{B}_{\mathbf{h}}^{\mathbf{m}} \hat{\mathbf{r}}\right) .
\end{aligned}
$$

Since the third term is purely radial, the approximation of eq. (7) conforms to the term of eq. (4), showing that the PM mode is primarily generated by tangentially divergent flow $\nabla_{h} \psi$, while the solenoidal flow $\hat{\mathbf{r}} \times \nabla_{h} \phi$ is the primary source of the TM mode. Note that $\nabla_{h} \phi$ also contributes part of the vertical electric source current. This is consistent with results given in Appendix D of Chave \& Luther (1990).

In this paper we select two regions to demonstrate the relation between tidal flow and the OTEM field: the northeastern Pacific (20$\left.52^{\circ} \mathrm{N}, 170^{\circ} \mathrm{E}-120^{\circ} \mathrm{W}\right)$ and Indian Ocean $\left(12-60^{\circ} \mathrm{S}, 40-102^{\circ} \mathrm{E}\right.$, see Fig. 1). The TPXO8 tide model shows that the average amplitude of M2 volume transport vector in the northeastern Pacific $\left(100 \mathrm{~m}^{2} \mathrm{~s}^{-1}\right)$ is twice the global average $\left(52 \mathrm{~m}^{2} \mathrm{~s}^{-1}\right)$ but the elevation is low and the $\mathrm{M} 2$ wave rotates around an amphidromic point $\left(25^{\circ} \mathrm{N}, 150^{\circ} \mathrm{W}\right)$ where the M2 tidal amplitude is zero. The tidal flow in this area is thus dominated by the solenoidal vorticity field $\hat{\mathbf{r}} \times \nabla_{h} \phi$, hence the TM mode would be the principle OTEM field on the ocean floor in the northeastern Pacific. In contrast, the M2 tide in the Indian Ocean is almost a standing wave with high elevation, and the gradient term $\nabla_{h} \psi$ dominates the tidal velocity field. In the Indian Ocean, $B_{r}^{m}$ is twice as strong as $B_{h}^{m}$, so the first term in eq. (7) outweighs the last term, and the OTEM field there would mainly be the PM mode.

\subsection{Theoretical sensitivity analysis}

Driven by different types of electric source current, the TM and PM modes exhibit different sensitivities to the subsurface resistivity structure. Considering the period and horizontal wavelength of the tides, the OTEM field is most sensitive to the resistivity of the oceanic lithosphere and underlying asthenosphere above the transition zone. Here we adopt two theoretical approaches to analyse the sensitivity of the TM and PM mode in a 1-D layered resistivity 

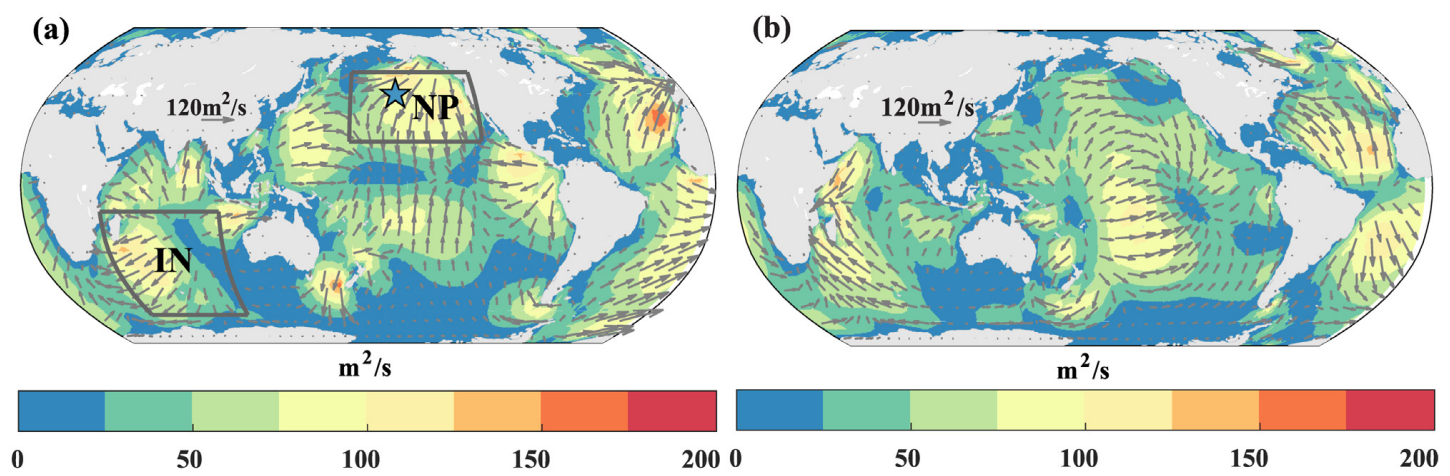

Figure 1. The tidal volume transport vector $\mathbf{V}$ of M2 constituent predicted by TPXO8 (Egbert \& Erofeeva 2002). The grey arrows denote the current. (a) The in-phase component. Areas labeled as 'NP' and 'IN' denote the northeastern Pacific and Indian Ocean that studied in the main text. The blue pentagram denotes the location of BEMPEX seafloor EM array. (b) The quadrature component.

model. First, we examine several key parameters of the reflection coefficients used to define the Green functions (Chave \& Luther 1990). Second, we study the semi-analytical solutions for the TM and PM modes in this simplified 1-D induction problem. In subsequent sections we use 3-D numerical modelling to verify and also explore the limitation of this analysis.

According to the reflection coefficients defined by Chave \& Luther (1990), the influence of electrical properties in the $j$ th layer (resistivity $\rho_{j}$ and thickness $h_{j}$ ) can be expressed by several parameters. In the TM mode, the influence is expressed by the admittance parameters $R_{j}$ and $r_{j}$ :

$R_{j}=\operatorname{coth}\left(\beta_{j} h_{j}\right) /\left(\beta_{j} \rho_{j}\right)$

$r_{j}=1 /\left(\beta_{j} \rho_{j}\right)$.

In the PM mode, it can be expressed by inductance parameters $Q_{j}$ and $q_{j}$ :

$Q_{j}=\mu \operatorname{coth}\left(\beta_{j} h_{j}\right) / \beta_{j}$

$q_{j}=\mu / \beta_{j}$

in which $\beta_{j}$ describes the effect of self induction in seawater and is defined as:

$\beta_{j}^{2}=\eta^{2}+\xi^{2}-i \omega \mu / \rho_{j}$,

where $\eta$ and $\xi$ are the zonal and meridional wavenumber of the tidal flow. For the M2 tide, the period is $12.42 \mathrm{hr}$ and the characteristic wavelength $\lambda \simeq 2000 \mathrm{~km}$. Assuming $\eta^{2}+\xi^{2}=(2 \pi / \lambda)^{2}$, eq. (12) can be numerically approximated as:

$\beta_{j}^{2} \sim 10^{-11}-2 \times 10^{-10} i / \rho_{j}$,

where the units of $\beta_{j}$ and $\rho_{j}$ are $\mathrm{m}^{-1}$ and $\Omega \mathrm{m}$, respectively. For the sake of simplicity, the approximate formulas in the follow discussion are purely numerical and the units of variables are SI basic units when they are not specified.

In a resistive layer $\left(\rho_{j}>1000 \Omega \mathrm{m}\right),\left|\beta_{j}\right|$ is almost constant $\left(\simeq 3.3 \times 10^{-6} \mathrm{~m}^{-1}\right)$. For an oceanic lithosphere $\left(50 \mathrm{~km} \leq h_{j}\right.$ $\leq 150 \mathrm{~km}), \operatorname{coth}\left(\beta_{j} h_{j}\right)$ can be approximated by $\left(\beta_{j} h_{j}\right)^{-1}$, hence $R_{j} \simeq 1 /\left(\beta_{j}^{2} \rho_{j} h_{j}\right) \sim 10^{11} /\left(\rho_{j} h_{j}\right)$. Since it is at least 2.2 times larger than $r_{j}, R_{j}$ would control the potential function of the TM mode. In other words, the TM mode is dependent on the transverse resistance $\rho_{j} h_{j}$ of the layer. Using the same approach, the lithosphere's influence on the PM mode can be numerically expressed by $Q_{j} \sim 10^{5} / h_{j}$, suggesting that the resistivity of lithosphere can hardly affect the PM mode, but the LAB depth might.

In a conductive $\left(\rho_{j}<20 \Omega \mathrm{m}\right)$ layer, $\beta_{j}^{2} \sim-2 \times 10^{-10} i / \rho_{j}$. If the thickness of this layer is rather large $\left(h_{j}>100 \mathrm{~km}\right)$, both $Q_{j}$ and $q_{j}$ can be numerically approximated by $0.1 \sqrt{\rho_{j}}$. In this case, the PM mode is closely related to the resistivity of the layer. The typical resistivity of the asthenosphere $\rho_{a}$ is $1-100 \Omega \mathrm{m}$, which means the relative magnitude of the two terms in eq. (13) cannot be easily decided, so the resistivity of the asthenosphere would possibly affect both TM and PM modes.

Next, we use a simple numerical model to compute the EM field for PM and TM modes with a single horizontal wavenumber in 1-D layered resistivity models in Cartesian coordinates. The modal response function can be defined as the ratio of potential to its vertical derivative on the surface: $\mu \Psi / \partial_{z} \Psi$ in the PM mode and $\sigma \Pi / \partial_{z} \Pi$ in the TM mode (Chave \& Weidelt 2012). The modal response function is not affected by the source and only contains information about the underground resistivity structure. The Maxwell equations for a 1-D model are simplified as two boundary value problems for independent modal functions. By using Dirichlet boundary condition on Earth's surface, we can obtain the independent poloidal and toroidal magnetic field. In our study, the period of M2 tide $T$ $\simeq 12.42 \mathrm{hr}$ and shallow water wavelength $\lambda=\sqrt{g H_{\text {sea }}} / T$ where $g=9.8 \mathrm{~m} \mathrm{~s}^{-2}$ and $H_{\text {sea }}$ is the seawater depth used in the computation. Assuming the horizontal wavenumber of the EM field is in the $y$-direction and the layered resistivity structure varies in the $z$ direction, the impedance $E_{x} / B_{y}$ is equivalent to the PM response function, and the admittance $B_{x} / E_{y}$ to the TM response function (up to multiplication by a constant, independent of Earth's resistivity).

As shown in Fig. 2, the 1-D solid earth model consists of a lithosphere with uniform resistivity $\rho_{l}$ and thickness $H_{l}$, and a conductive asthenosphere extending from $H_{l}$ to the top of the transition zone with resistivity $\rho_{a}$, and two underlying mantle layers with constant resistivity of $10 \Omega \mathrm{m}(410-660 \mathrm{~km})$ and $3 \Omega \mathrm{m}(660-760 \mathrm{~km})$. The resistivity of the asthenosphere $\rho_{a}$ varies with the depth, assuming a simple thermal profile with a mantle potential temperature of $1350^{\circ} \mathrm{C}$ and an adiabetic gradient of $0.3{ }^{\circ} \mathrm{C} \mathrm{km}^{-1}$. We parametrize resistivity in the asthenosphere through a dependence on water content $c w$, with $\rho_{a}$ estimated following the experimental study of Dai $\&$ Karato (2014) on hydrated olivine. In this section, the range of $c w$ is $10-200 \mathrm{ppm}$, corresponding to depth-averaged $\rho_{a}$ of $240-22$ $\Omega \mathrm{m}$.

Fig. 3 shows contour plots of PM and TM response function (normalized amplitudes) with varying $\rho_{l}, \rho_{a}$ and $H_{l}$ in logarithmic axes. Solid and dashed contours illustrate changes due to variations 


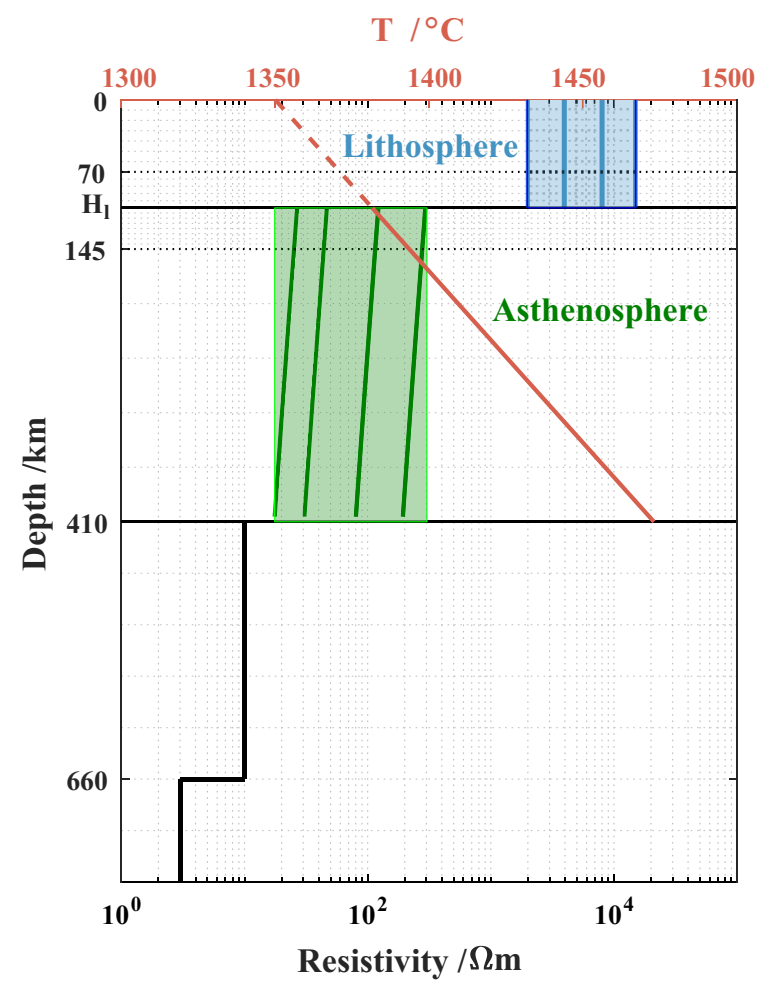

Figure 2. The thermal (red line) and resistivity profile of 1-D global models. The blue lines denote the resistivity of the lithosphere $(2000,4000,8000$ and $1.5 \times 10^{4} \Omega \mathrm{m}$ ), and green ones are for the resistivity of asthenosphere (the water content is 10,30,100 and $200 \mathrm{ppm}$ ). The LAB depth $H_{l}$ (upper grey line) varies between 70 and $145 \mathrm{~km}$ depth (dashed grey lines).

in the third model parameter (i.e. $\rho_{a}$ in top row, resistance $H_{l} \rho_{l}$ in bottom). In Fig. 3(a), the solid contours $\left(\rho_{a}=50 \Omega m\right)$ are nearly vertical, implying that the PM mode is barely influenced by $\rho_{l}$, while $H_{l}$ would moderately change the amplitude. Even though the dotted contours $\left(\rho_{a}=200 \Omega m\right)$ flex more when the lithosphere is less resistive, the variation might be too small to be useful. By contrast, the linear contours in Fig. 3(b) are parallel to the line $\rho_{l} H_{l}$ $=$ const (black dashed line), and the solid $\left(\rho_{a}=50 \Omega m\right)$ and dotted $\left(\rho_{a}=200 \Omega m\right)$ lines are indistinguishable when the lithosphere is very resistive. Thus, the TM mode amplitude is primarily controlled by the transverse resistance $\left(\rho_{l} H_{l}\right)$ of the lithosphere, especially when $\rho_{l}$ is large.

To further clarify the influence of the LAB depth on the TM mode, Figs 3(c) and (d) plot amplitude variation for fixed lithospheric resistance. See the caption of Fig. 3. The PM mode displays a high sensitivity to $\rho_{a}$. It should also be noted that when $\rho_{a}<50 \Omega \mathrm{m}$, the influence of the LAB depth is enhanced. The solid $\left(H_{l} \rho_{l}=8 \times\right.$ $\left.10^{8} \Omega \mathrm{m}^{2}\right)$ and dotted $\left(H_{l} \rho_{l}=4 \times 10^{8} \Omega \mathrm{m}^{2}\right)$ contours are almost identical, further demonstrating that lithospheric resistivity has little effect on the PM mode. The significant difference between the solid and dotted contours in Fig. 3(d) indicates the strong dependence of the TM mode on integrated lithosphere resistance. By comparison, the asthenospheric resistivity $\rho_{a}$ and the LAB depth only have minor effects on the TM mode. Comparable results for phase are shown in Fig. S2 in supplementary material; these plots contain similar information to the amplitude plots shown here.

These results agree with our analysis of the reflection coefficients. The TM mode is primarily sensitive to the transverse resistance, while the PM mode can better constrain the resistivity of the conducting asthenosphere. The PM mode is also sensitive to the $\mathrm{LAB}$ depth when the asthenosphere is conductive enough. The simplified theoretical analysis presented here aims to provide a general insight into the EM modes, which is related to observation technique (satellite or seafloor). Note that even though the OTEM fields above/in the deep ocean are generally consistent with the simplified theoretical analysis, but in a more realistic earth model, inhomogeneity of the conductance of the surface layer (ocean and continent) and electric source current would add complexity to the induced EM field in coastal areas, hence the analysis may not be valid any longer and its direct application to scenarios with realistic ocean bathymetry and sources can be misleading. In the following section, we model OTEM fields by solving the 3-D induction problem with a realistic heterogeneous internal electric source current, allowing full coupling between EM modes.

\section{NUMERICAL METHOD}

To simulate the OTEM field in a more realistic Earth and ocean model, we use a new 3-D forward model based on the FD method, with eq. (1) discretized on a staggered grid in spherical coordinates (Fig. 4). The computational domain includes the overlying resistive atmosphere and the conductive ocean and Earth which are discretized with prisms of homogeneous resistivity. Derivation of the coefficient matrix for the system of equations is similar to Egbert \& Kelbert (2012), except that metric elements appropriate for spherical coordinates are used (see also Uyeshima \& Schultz 2000).

Our implementation allows for a full global grid, and nested higher-resolution local spherical-coordinate grids. For global modelling, the forcing within ocean layers and simple homogeneous Dirichlet boundary condition are specified, that is the tangential electric fields are zero on the upper and lower spherical boundaries. For nested regional modelling, boundary data are also required for lateral boundaries. Tangential fields on these boundaries are obtained from interpolation of the global solution. For both global and local model the linear equations are solved iteratively using the bi-conjugate gradient stabilized (BiCGstab) method with an incomplete LU (ILU) preconditioner. A divergence correction is applied periodically to the sum of conduction current and electric source current to eliminate spurious solutions and accelerate convergence (Mackie et al. 1994; Uyeshima \& Schultz 2000).

In this study, the electric source current $J_{s}$ is calculated following eq. (2) with $M_{2}$ tidal currents from the $0.167^{\circ} \times 0.167^{\circ}$ resolution data assimilating global barotropic tide model TPXO8 (Egbert \& Erofeeva 2002), 3-D seawater conductivity dataset derived from World Ocean Atlas 2013 (Tyler et al. 2017), and the ambient geomagnetic field model IGRF-12 (Thébault et al. 2015). The computation domain includes a $10^{6}$-km-thick overlying atmosphere and extends to a depth of $760 \mathrm{~km}$ in the conductive Earth. A realistic ocean bathymetry and sediment thickness map (Whittaker et al. 2013) is incorporated to define resistivity variations in ocean basins. Horizontal grids are uniform, with spacing for global and local model $2^{\circ} \times 2^{\circ}$ and $0.2^{\circ} \times 0.2^{\circ}$, respectively. The radial grid spacing starts from $50 \mathrm{~m}$ at Earth's surface and expands upwards (and downwards) as a geometric series increasing by a factor of 1.2, except that the lower part of the ocean layer at 2-5 km depth uses constant $200 \mathrm{~m}$ vertical spacing.

In this section, the 1-D solid earth model is similar to the models illustrated in Fig. 2 except that the upper mantle between the LAB depth and transition zone has uniform resistivity $\rho_{a}$. The default global model with $\rho_{l}=8 \times 10^{3} \Omega \mathrm{m}, H_{l}=70 \mathrm{~km}$ and $\rho_{a}=100 \Omega \mathrm{m}$ is used when parameters are not specified. We follow the right-hand 

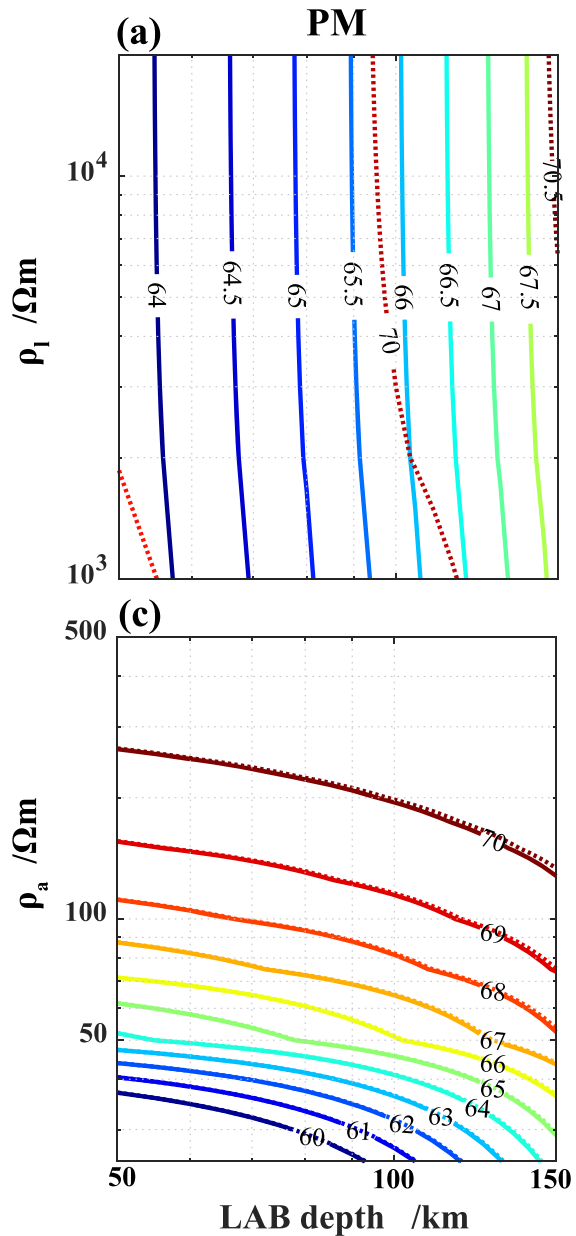

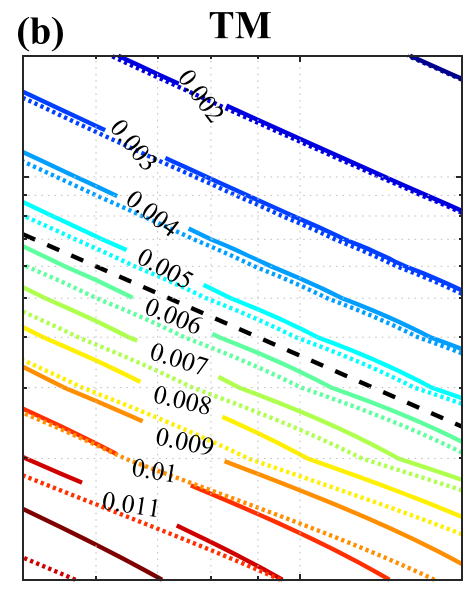

(d)

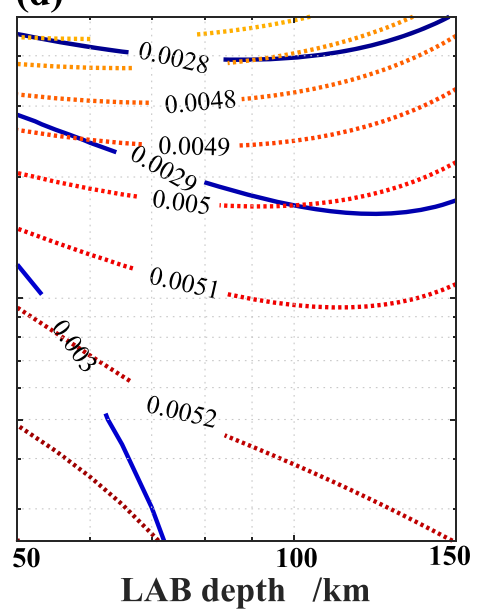

Figure 3. Contour plot of amplitude of PM (left-hand column) and TM (right-hand column) response function varying with (a) and (b) lithospheric resistivity $\rho_{l}$ and the LAB depth. The solid and dotted contours are for models in which $\rho_{a}$ is 50 and $200 \Omega \mathrm{m}$, respectively. The black dashed line in b denotes the line $\rho_{l} H_{l}=$ const. (c)-(d) asthenospheric resistivity $\rho_{a}$ and the LAB depth with fixed lithospheric resistance $\rho_{l} H_{l}$. The solid and dotted contours are for models in which the resistance is $8 \times 10^{8}$ and $4 \times 10^{8} \Omega \mathrm{m}^{-2}$, respectively.

(a)

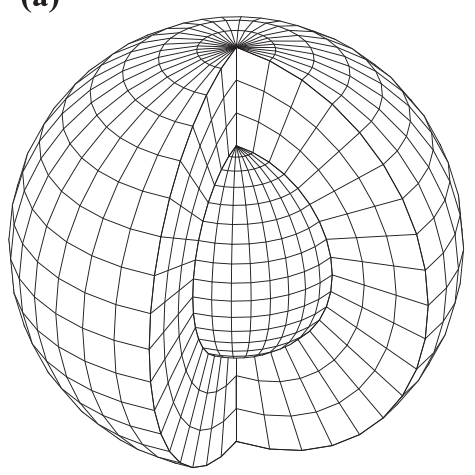

(b)

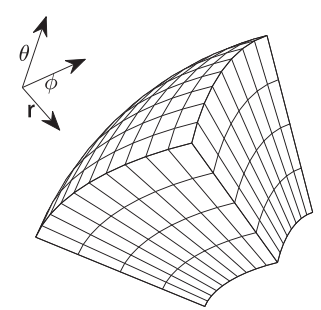

Figure 4. Sketch of the mesh division in spherical coordinates for (a) a global model and (b) a regional model.

rule for all coordinate systems, and use the Cartesian coordinate symbols $x, y$ and $z$ for northward, eastward and downward components in place of $\theta, \phi$ and $r$ to denote local spherical coordinates.

Modelling results suggest that the vertical magnetic field $B_{z}$ at satellite altitude $(430 \mathrm{~km})$ and the north magnetic field $B_{x}$ on the seafloor are representative components (dominated by PM and TM modes, respectively). These are illustrated in Fig. 5. Even though

difference in amplitudes do exist, the main spatial feature of the predicted satellite $B_{z}$ agrees with the time-dependent geomagnetic field model CM5 (Sabaka et al. 2015; see Fig. S3) and M2 signals from Swarm satellite observation (Sabaka et al. 2016; Fig. 2).

Fig. 5 shows that the distribution of OTEM fields is strongly influenced by the geomagnetic background field and the ocean tidal volume transport vector which define the source currents. The purely PM satellite OTEM signal exhibits large amplitude above regions with both strong radial geomagnetic field and tangentially divergent flow, including the central Indian Ocean, offshore New Zealand and the northeastern Atlantic Ocean. At the same time the satellite OTEM field is rather small near the geomagnetic equator where source current for the PM mode should vanish (based on the 1-D analysis). Seafloor horizontal magnetic fields with large amplitude occur in some of the same regions noted above, but also occur in other areas, such as the Northeastern Pacific. Here the amplitude of seafloor $B_{x}$ is up to $5 \mathrm{nT}$ while satellite $B_{z}$ is merely $0.5 \mathrm{nT}$, much less than the average value above the Indian Ocean. As discussed in Section 2.1, this phenomenon can be explained by the relation between the tidal flow and modes of OTEM field. The PM mode, driven primarily by the irrotational (poloidal) component of tidal flow, dominates in the Indian Ocean and other regions with strong $B_{z}$. The strong PM magnetic signal can exist in both the insulating air and conductive Earth, so large amplitudes could be observed 


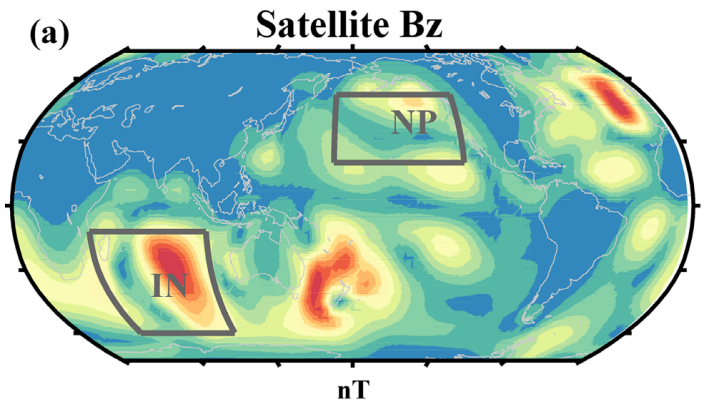

nT

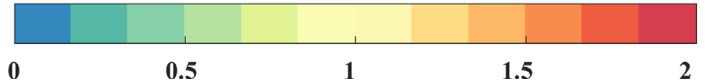

(b)

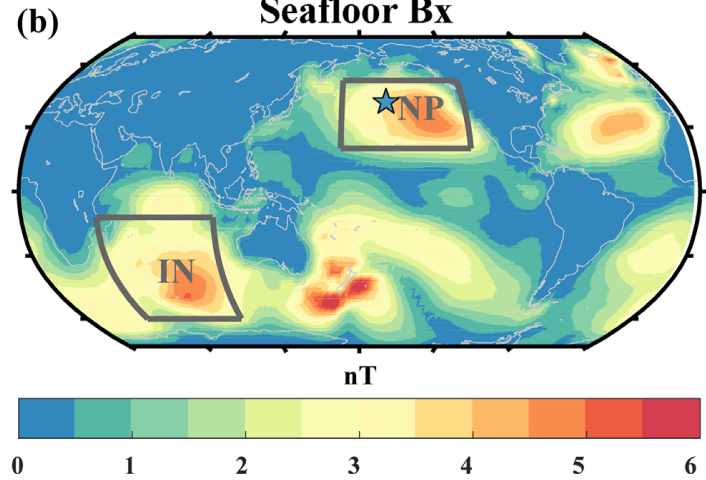

Figure 5. The amplitude of the M2 OTEM field. (a) The vertical magnetic component $B_{z}$ at satellite altitude (430 km). (b) The north magnetic component $B_{x}$ on the seafloor. Other symbols are same with Fig. 1.
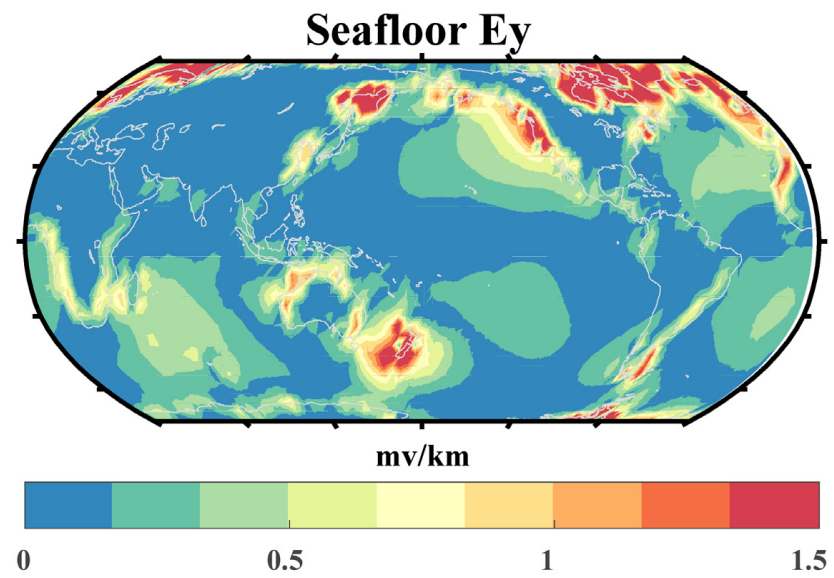

Figure 6. The amplitude of east electric field $E_{y}$ on the seafloor.

both at the satellite height and on the seafloor. In contrast, the TM mode, which is generated by the vorticity-like flow and not measurable in the air, makes up most parts of the seafloor horizontal magnetic field in the northeastern Pacific. Since most of the energy generated by solenoidal tidal flow (TM mode) is kept within the conductive Earth and ocean, large amplitude could be seen on the seafloor in northeastern Pacific. See Fig. S4 for the phase of satellite $B_{z}$ and seafloor $B_{x}$.

While many of the features seen in Fig. 5 can be explained by the 1-D theory of Section 2, the continent-ocean resistivity contrast will distort and couple the primary poloidal/toroidal magnetic fields and produce secondary toroidal/poloidal fields. Secondary poloidal magnetic fields can be recorded by satellites and onshore observatories, and may contain partial information about the TM mode. The decrease in water depth (which amplifies tidal currents) and sharp resistivity contrast between ocean and continent results in a strong amplification of seafloor electric fields (up to $2 \mathrm{mV} / \mathrm{km}$ ) in some coastal areas (Fig. 6), especially around the margins of the North Pacific and North Atlantic, around Australia and New Zealand and on the Patagonian Shelf. All of these areas exhibit strong alongshore tidal currents, that can be modelled as large scale Kelvin waves. Analysis of an idealized Kelvin wave model demonstrates that the seafloor electric field is governed most directly by the electric source current term (Chave 1983), and hence will be more useful for monitoring ocean flows (Saynisch et al. 2017), rather than the resistivity beneath the ocean. However, in our modelling results the strong electric fields frequently extend well onshore. In fact, details of these coastal electric fields will be significantly affected by the degree of leakage of electric currents into the mantle at subduction zones (Evans et al. 2002), and tide-induced electric field observed near continental margins may be useful for studies of Earth resistivity. Because the focus of this study is on use of OTEM data to study mantle resistivity beneath the ocean basins, we mainly discuss the magnetic field in following sections.

\section{ERRORS FROM THE UNCERTAINTY IN TPXO}

In addition to Earth's resistivity structure, the OTEM field depends on the tidal electric current source $\mathbf{J}_{\mathbf{s}}$. All of the inputs required to calculate $\mathbf{J}_{\mathbf{s}}$ (see eq. 2) are imperfectly known, and an assessment of how these uncertainties propagate to errors in computed OTEM fields is necessary. Grayver et al. (2016) discussed the uncertainty in seawater conductivity $\sigma_{s}$ and ambient geomagnetic field $\mathbf{B}^{\mathbf{m}}$, so we focus here on the impact of uncertainties in tidal currents $\mathbf{v}$. Our analysis builds on the statistical model implicit in the data assimilation formalism used for computing tidal currents in the TPXO model series (Egbert et al. 1994). It is very similar to the Monte Carlo analysis of errors in tidal currents and tidally induced gravitational perturbations, as presented by Dushaw et al. (1997) and Ray et al. (2001), respectively. We summarize the procedure here.

The tidal current fields in TPXO8 are not directly observed, but rather are derived from assimilation of multi-mission radar altimeter data into a simplified hydrodynamic model [essentially the Laplace Tidal Equations, or LTE; see (Egbert et al. 1994), and (Egbert \& Erofeeva 2002), for further details]. The assimilation is formulated as a stochastic inverse problem, with estimates of prior errors in the LTE derived from analysis of errors due to approximations in the equations, bathymetry errors, and numerical grid truncation (Egbert et al. 1994). These are treated as errors in tidal forcing, which can be used together with estimates of observational errors to compute posterior errors for estimated tidal fields. The LTE are solved with the known astronomical forcing perturbed with a series of $N$ error fields, derived according to the prior error covariance, to obtain synthetic tidal velocity fields $\mathbf{v}_{n}, n=1, N$. The corresponding tidal elevation fields are then sampled with the spatial and temporal patterns of the altimeter, and the resulting synthetic data (with random noise representative of observational error and non-tidal oceanography added) are inverted. The same procedure applied to the real data is used, to obtain estimated velocity fields $\hat{\mathbf{v}}_{n}, n=1, N$. Differences $\mathbf{v}_{n}-\hat{\mathbf{v}}_{n}, n=1, N$ are representative of errors in the actual assimilated velocity field, that is the covariance of the difference 
fields is the posterior covariance. Although the error covariance scheme is a (rather ad hoc) comparison to independent validation data in previous applications (Dushaw et al. 1997; Ray et al. 2001), the procedure provides reasonable estimates of error variances.

In this study, errors in M2 velocities are obtained using $N=40$ Monte Carlo realizations. Propagation of error in velocity to error in the OTEM fields is straightforward. Owing to the linearity of the source term, the numerical solution of eq. (1) with the RHS calculated using the tidal velocity error realizations (i.e. $\mathbf{v}_{n}-\hat{\mathbf{v}}_{n}$ ), yields a sample of OTEM error fields. Pointwise root mean square (RMS) of these computed error fields provide an estimate of the impact of tide model uncertainties on the OTEM field predictions.

The RMS value of fluctuations in OTEM fields from 40 forward modelling runs is illustrated in Fig. 7. In general, errors are large in coastal areas, and negligibly small in the ocean interior. The error in the modelled vertical magnetic component $B_{z}$ at satellite altitudes is up to $0.03 \mathrm{nT}$ along the margins of the North Atlantic, south of the Aleutian Arc, in the South China Sea and offshore New Zealand. Modelled values in these areas are 1-2 nT, so this represents a relative error of at most 5 per cent, and generally less. The error in the seafloor north magnetic component $B_{x}$ is larger than $0.15 \mathrm{nT}$ in the same areas, but again of the order of 5 per cent or less for relative error. In most of the ocean interior, errors in modelled satellite and seafloor magnetic fields are less than 0.01 and $0.05 \mathrm{nT}$, respectively. Relative errors are generally less than 2 per cent, and often below 1 percent. As we show in the next section (see Fig. 8), moderate variations in the resistivity of lithosphere/mantle would result in fluctuations of the magnetic fields with a magnitude of $0.1-0.5 \mathrm{nT}$. Thus, while uncertainty in the tidal velocities has a small (if not always negligible) impact in the ocean interior, in particular coastal areas (e.g. offshore New Zealand), the errors can be comparable to plausible signals due to Earth's structure.

\section{SENSITIVITY TEST}

In this section we utilize 3-D numerical modelling to further investigate the sensitivity of OTEM fields to Earth's resistivity structure. The three-parameter 1-D model described in Section 2.2 (also see Fig. 2) is used. We consider four values for lithospheric resistivity $\rho_{l}: 2000,4000,8000$ and $1.5 \times 10^{4} \Omega \mathrm{m}$, six values for LAB depth $H_{l}: 70,85,100,115,130$ and $145 \mathrm{~km}$, and four values for water content $c w: 10,30,100$ and $200 \mathrm{ppm}$, which with our assumptions correspond to average asthenospheric resistivity $\rho_{a}$ of $240,100,40$ and $22 \Omega \mathrm{m}$ (decreasing with increasing water content). We thus conduct fully 3-D simulations for a total of 96 global 1-D resistivity profiles, all overlain by realistic ocean bathymetry and sediment layer.

Fig. 8 illustrates the variations in the amplitudes of the satellite and surface $B_{z}$ and seafloor $B_{x}$ components caused by changes in $H_{l}$ and $c w$. See Fig. S5 in the supplementary material for the phases, which exhibit similar patterns. The default (reference) value of model parameters is $\rho_{l}=8000 \Omega \mathrm{m}, H_{l}=70 \mathrm{~km}$ and $c w=100 \mathrm{ppm}$ $\left(\rho_{a}=40 \Omega \mathrm{m}\right)$.

The left-hand column of Fig. 8 illustrates the impact of increasing $H_{l}$ from 70 to $130 \mathrm{~km}$. At satellite altitude (Fig. 8a), large variations in $B_{z}$ up to $0.1 \mathrm{nT}$ are generally seen in the same areas where amplitudes are greatest in Fig. 5. In most cases $B_{z}$ increases with a deeper LAB depth, especially for patches above the ocean interior. The surface $B_{z}$ component (Fig. 8c) exhibit similar response but with larger amplitude ( $0.2 \mathrm{nT}$ colour scales in Figs $8 \mathrm{a}-\mathrm{b}$ and $\mathrm{c}-\mathrm{d}$ are different). This is consistent with the analytical analysis as shown in Fig. 3(a) that the amplitude of the poloidal response function increases with the LAB depth. But in some near-coastal regions, including the Northeastern Pacific, Eastern North America, Europe, Southwest Africa and Australia, the amplitude decreases, which results from the coupled PM and TM modes (secondary PM field) caused by the continent-ocean resistivity contrast in a realistic 3-D model with coastlines. On the seafloor(Fig. 8e), the amplitude of $B_{x}$ exhibits changes exceeding $0.5 \mathrm{nT}$, dominantly with the amplitude decreasing for larger $H_{l}$. These large reductions in amplitude (in the northeastern Pacific, southern Indian, and north and south Atlantic oceans, and around New Zealand) are all in regions with a strong $B_{x}$ signal (Fig. 5). This trend toward decreased amplitude with larger $H_{l}$ is consistent with the analytic results for the TM mode shown in Fig. 3(b). There are also very large increases in amplitude in areas adjacent to the high-amplitude patches, especially along the magnetic equator in the Pacific. The complex pattern of changes in these plots is a clear demonstration of the need for 3-D interpretation of the OTEM fields.

The right-hand column of Fig. 8 shows that when the mantle water content increase from 100 to $200 \mathrm{ppm}$ (average $\rho_{a}$ decreases from 40 to $22 \Omega \mathrm{m}$ ), the amplitude of the satellite and surface $B_{z}$ component decreases, and the seafloor $B_{x}$ component increases. Above the Indian ocean, New Zealand and north Atlantic ocean, where the PM mode magnetic field is dominant, the decrease is roughly 0.2 and $0.3 \mathrm{nT}$ in the amplitude of the satellite (Fig. $8 \mathrm{~b}$ ) and surface $B_{z}$ (Fig. 8d), and the relative change is more than 10 per cent. In regions mentioned above and northeastern Pacific, the increase in the seafloor $B_{x}$ is roughly $0.3 \mathrm{nT}$ (Fig. 8f). The amplitude of seafloor $B_{x}$ is larger than that of the surface $B_{z}$ in most regions mentioned above, so the relative change of seafloor $B_{x}$ is smaller than that of surface/satellite $B_{z}$. This feature agrees with the theoretical analysis that the amplitude of the poloidal magnetic field decreases with the resistivity of the asthenosphere, and the toroidal magnetic field varies in the opposite way and exhibits less sensitivity (Figs $3 \mathrm{c}$ and d). It also suggests that much (but not all) of seafloor $B_{x}$ is TM mode.

The comparison between the first and second row of Fig. 8 demonstrates that $B_{z}$ at satellite altitude and on Earth's surface show similar response to variations in earth model parameters, except that the surface field has a larger amplitude because it is closer to tidal electric source current. Since satellite and surface OTEM fields have the same physical nature (the PM mode), for the remainder of this study, we only focus on the satellite $B_{z}$ and seafloor $B_{x}$.

Nevertheless, the OTEM field's capability for probing deep Earth has limitations. As illustrated in Fig. 9(a), if $H_{l}$ increases from 70 to $100 \mathrm{~km}$ while $\rho_{a}$ decreases from 60 to $40 \Omega \mathrm{m}$, there is no measurable change above the ocean interior in satellite observations. This trade-off between the resistivity and upper boundary depth of a conductive layer resolved by satellite field corresponds to the feature of PM mode which is mostly influenced by $\rho_{a}$ and $H_{l}$. In Fig. 9(b), the variation in the seafloor $B_{x}$ amplitude is below 0.1 nT when the lithosphere becomes both less resistive and thicker $\left(\rho_{l}=4 \times 10^{3} \Omega \mathrm{m}, H_{l}=150 \mathrm{~km}\right)$. Since the strong dependence of seafloor $B_{x}$ on $H_{l}$ mainly results from the sensitivity of the TM mode to transverse resistance (the product of $\rho_{l}$ and $H_{l}$ ), if this quantity were held constant while increasing LAB depth, variations in $B_{x}$ would be much more subtle than what would be caused by individual changes in $\rho_{l}$ and $H_{l}$.

Considering the spatial variation in tidal electric source current, we examine the Indian Ocean and northeastern Pacific (labeled regions in Fig. 1) in further detail. The OTEM signal in the default model $\left(\rho_{l}=8000 \Omega \mathrm{m}, H_{l}=70 \mathrm{~km}\right.$ and $\left.c w=100 \mathrm{ppm}\right)$ is the 

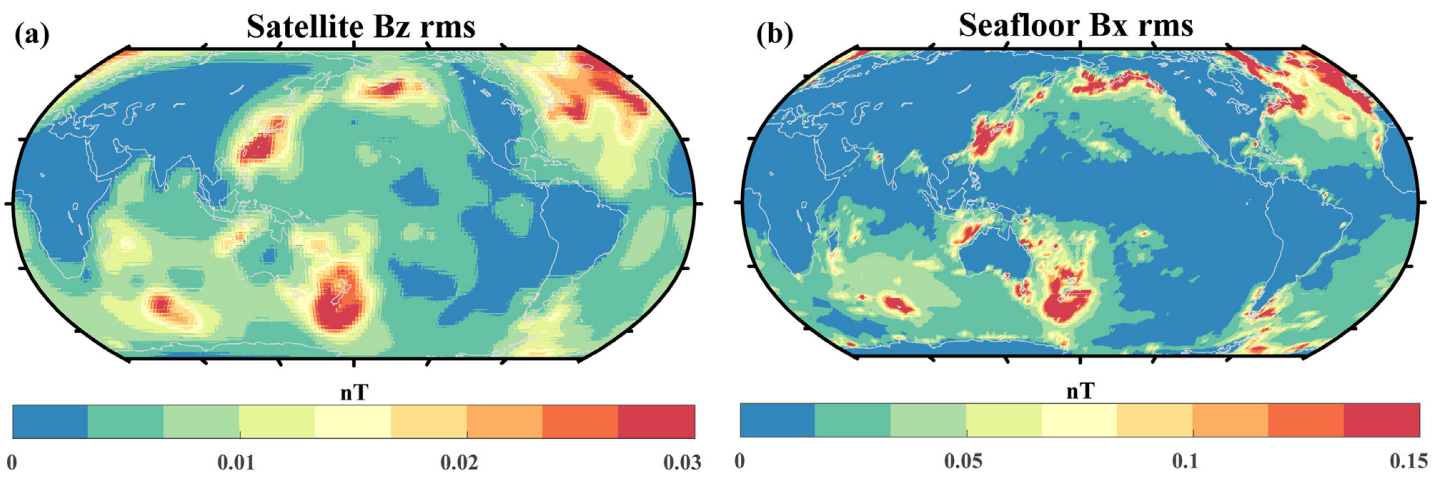

Figure 7. RMS value of the deviation in OTEM field caused by the uncertainty in TPXO8 M2 transport model. (a) The satellite $B_{z}$ component. (b) The seafloor $B_{x}$ component.
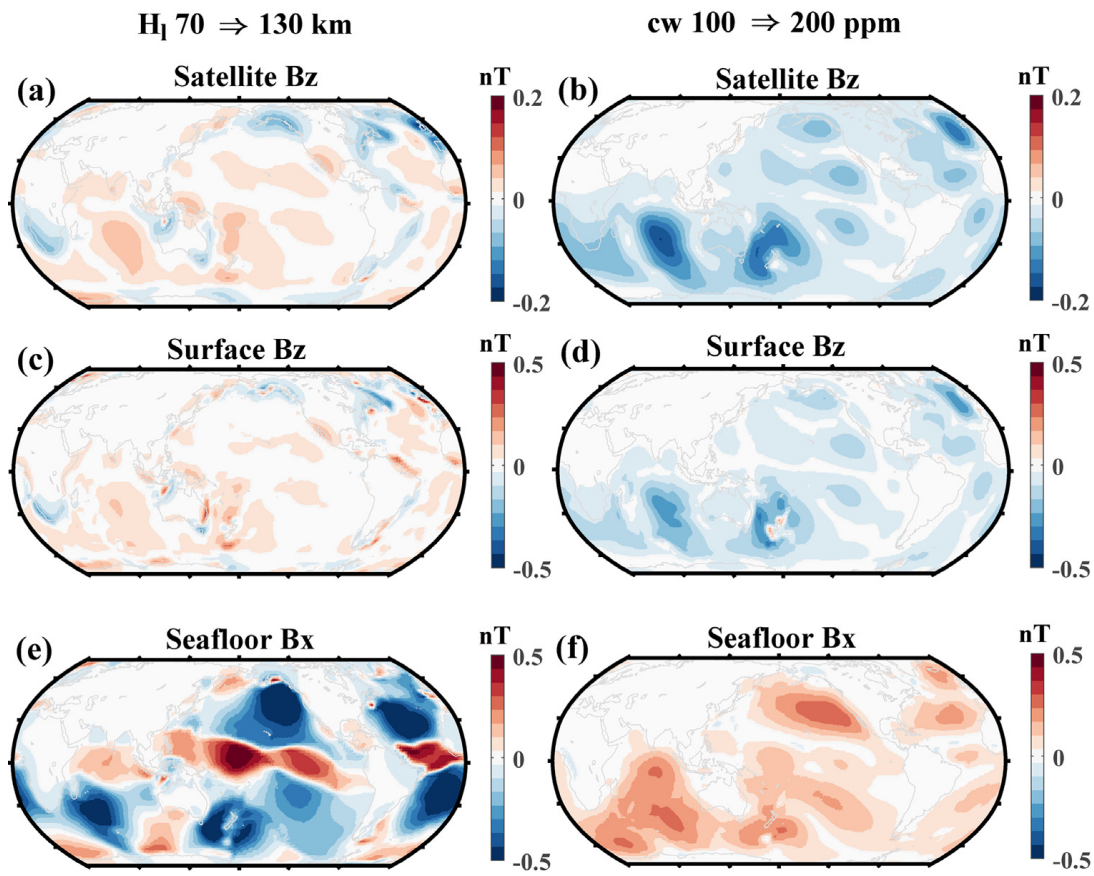

Figure 8. Changes in the amplitudes of magnetic signal when the LAB depth increases from 70 to $130 \mathrm{~km}$ (the left column), and the mantle water content increases from 100 to $200 \mathrm{ppm}$ (the right column). (a)-(b) The vertical magnetic signal $B_{z}$ at satellite altitude. (c)-(d) The vertical magnetic signal $B_{z}$ on the Earth's surface. (e)-(f) The north component $B_{x}$ on the seafloor. Note that different colour scales are used for satellite and seafloor/surface data.

\section{Surface Bz}

(a)

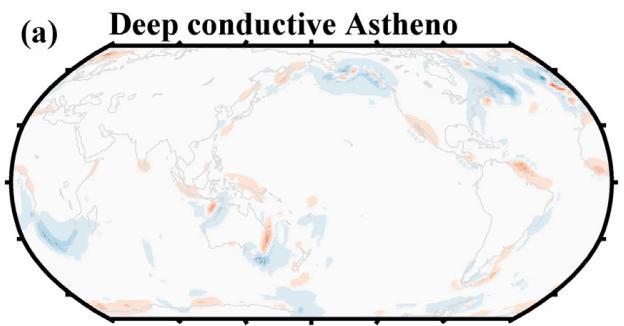

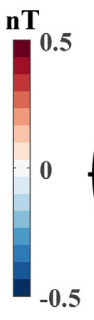

Seafloor Bx

(b) Thick conductive Litho

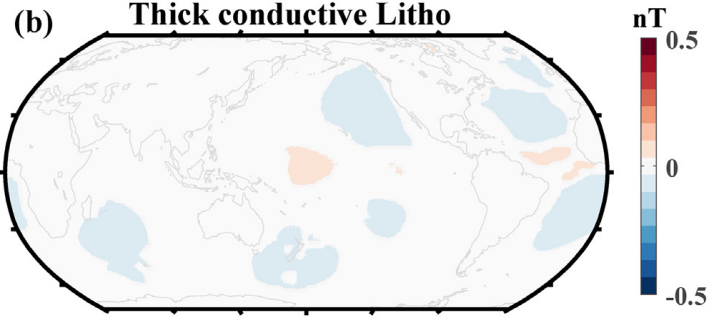

Figure 9. Changes in amplitude of (a) satellite $B_{z}$ if the LAB is deeper and the asthenosphere is more conductive. (b) Seafloor $B_{x}$ if the lithosphere (with fixed resistance) is thicker and less resistive.

reference field. For the other 95 model runs, the relative change of amplitude and absolute difference of phase between the modelling result and reference field are first weighted by the normalized amplitude of the reference field, then averaged over the spatial domain to generate the regional average change of the OTEM field. The relative change of amplitude (in percentage) and absolute difference of phase (in degree) are presented in Figs 10 and 11 as functions of the lithospheric resistance $\rho_{l} H_{l}$ and average resistivity of the 
IN satellite $\mathbf{B z}$
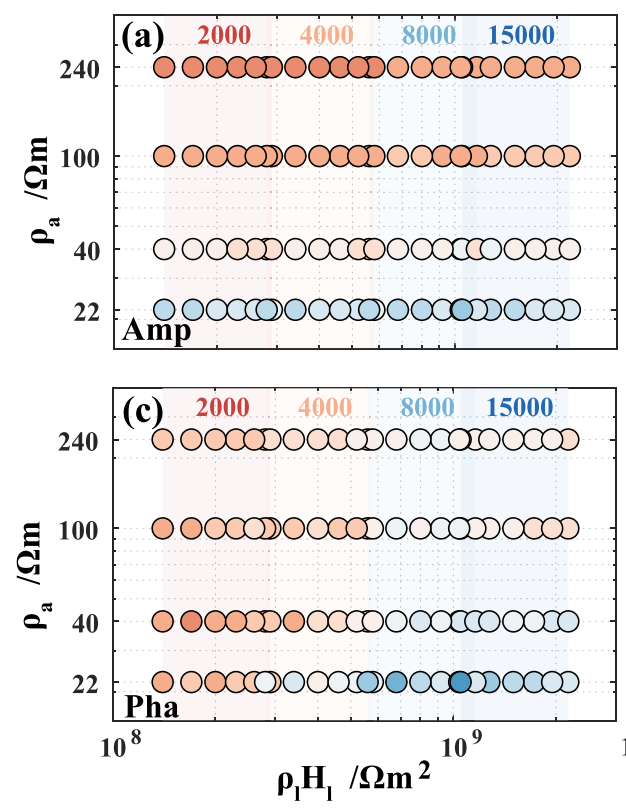

NP satellite Bz
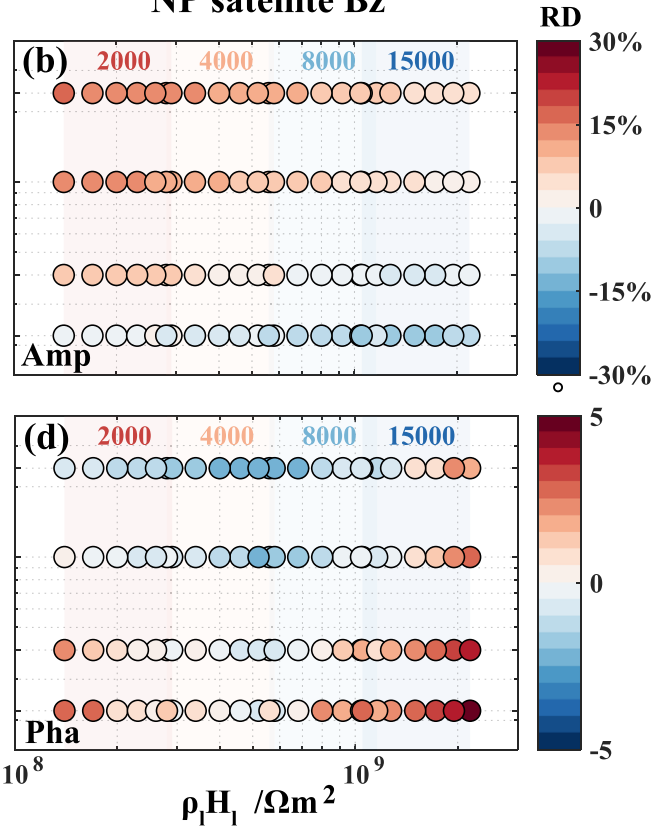

Figure 10. The pseudocolour plot of satellite $B_{z}$ component above the Indian Ocean (left-hand column) and northeastern Pacific (right-hand column) as a function of average resistivity of asthenosphere $\rho_{a}$ and lithospheric resistance $\rho_{l} H_{l}$. (a)-(b) The relative change of amplitude. (c)-(d) The absolute difference of phase. In each single plot, coloured circles are results extracted from different models, and shaded columns from left to right correspond to four values of $\rho_{l}$ labeled above (see main content).

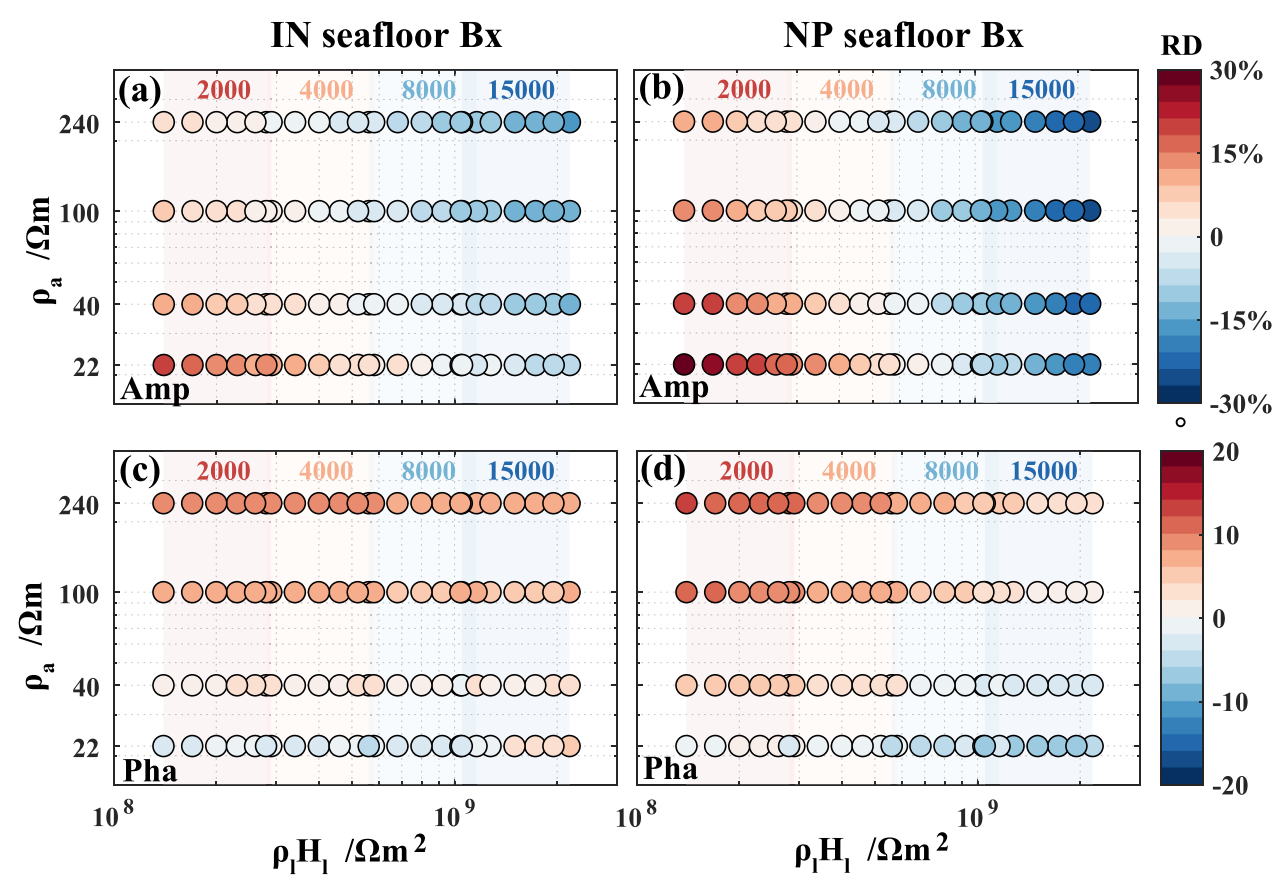

Figure 11. The pseudocolour plot of seafloor $B_{x}$ component in the Indian Ocean (left-hand column) and northeastern Pacific (right-hand column) as a function of $\rho_{a}$ and $\rho_{l} H_{l}$. (a)-(b) The relative change of amplitude. (c)-(d) The absolute differences of phase. Other symbols are similar to Fig. 10, but with a larger scale in colourbar.

asthenosphere $\rho_{a}$. Separate $\rho_{l}, H_{l}$ and $\rho_{a}$ can also be recognized (see the caption of Fig. 10).

The amplitude of the satellite $B_{z}$ component (at $430 \mathrm{~km}$ height) above the Indian Ocean increases with $\rho_{a}$, and is controlled by the asthenosphere, but the influence of the lithospheric parameters is more subtle (Fig. 10a). A decrease of $\rho_{a}$ by half (e.g. 40-22 $\Omega$ ) causes a 10 per cent reduction in satellite $B_{z}$ amplitude. Although amplitude shows very little sensitivity to lithosphere thickness or resistivity, phase is somewhat sensitive to $\rho_{l}$, increasing if the earth model is more conductive (Fig. 10c). No consistent pattern of phase 
variation is seen for change in lithospheric thickness. Above the northeastern Pacific, both the lithosphere and asthenosphere influence the satellite observations (Figs $10 \mathrm{~b}$ and d). The amplitude of satellite $B_{z}$ increases with $\rho_{a}$ and decreases with $\rho_{l}$, and seems to be controlled by the ratio $\rho_{l} / \rho_{a}$. There are also changes in phase associated with variations in LAB thickness. For example, doubling the LAB depth from 70 to $145 \mathrm{~km}$ would cause phase to decrease by approximately $2^{\circ}$ when $\rho_{a} \leq 40 \Omega \mathrm{m}$. However, the changes are subtle, and not always in the same direction.

For the seafloor $B_{x}$ component (Fig. 11), variations are generally larger (note difference in scale of phase with Fig. 10, especially in the northeastern Pacific). In both areas, lithospheric resistance $\rho_{l} H_{l}$ has the largest impact on amplitude, which decreases with increasing resistance. Doubling the lithospheric resistance $\rho_{l} H_{l}$ results in roughly a 20 per cent decrease in amplitude. Note that amplitude is also influenced by $\rho_{a}$ and $H_{l}$, especially when the asthenosphere is more conductive. On the Indian Ocean bottom (Fig. 11c), the phase of $B_{x}$ is mainly dependent on $\rho_{a}$, with a decrease by half (e.g. 100 to $40 \Omega$ ) causing a decrease in phase of more than $5^{\circ}$. By contrast, the $B_{x}$ phase on the northeastern Pacific follows a similar pattern to the amplitude of satellite $B_{z}$, varying with the ratio $\rho_{l} / \rho_{a}$. Decreasing this ratio by half results in an increase of $5^{\circ}$ in phase.

Figs 10 and 11 show that the OTEM data have different sensitivity to the resistivity model, depending on the observation method (satellite or seafloor), data type (amplitude or phase) and tidal flow type (divergent or solenoidal). The amplitude of the horizontal magnetic signal on the ocean bottom, which contains both the PM and TM modes, is very sensitive to the transverse resistance of the lithosphere $\rho_{l} H_{l}$, and is also influenced by the resistivity of the asthenosphere $\rho_{a}$ and the LAB depth $H_{l}$ if the asthenosphere is conductive enough. Nonetheless, the sensitivity of OTEM satellite data as well as the phase of seafloor data depends on the tidal flow type. For the Indian Ocean, where the tangentially divergent flow dominates the M2 tide, satellite data and the phase of seafloor data are sensitive to $\rho_{a}$, while for the northeastern Pacific, where the solenoidal flow is more important, the resistivity contrast between the lithosphere and asthenosphere, namely $\rho_{l} / \rho_{a}$, controls the satellite data and seafloor phase, and $H_{l}$ can influence the data as well if the asthenosphere is rather conductive.

In most cases, mixed flow types and secondary toroidal/poloidal fields make it less possible to quantitatively apply the simplified theoretical analysis or decomposition to real data. We recommend the combination of multiple OTEM observations. Taking the Indian Ocean as an example, the resistivity of the asthenosphere can be constrained by the amplitude of satellite data and the phase of seafloor data, and the lithospheric resistance can be constrained by the amplitude of seafloor data. Moreover, the $B_{z}$ component on the seafloor, which is PM mode, can also constrain the asthenosphere and the LAB depth (Fig. S6), since the OTEM field on the ocean bottom is closer to the resistivity anomaly and contains more magnetic signal at short wavelengths that may provide more detailed information about the resistivity structure. It should also be mentioned that subtle changes in the EM field might be difficult to resolve from observations that suffer from noise due to different sources, for example, a doubling of $\rho_{l}$ or $\rho_{a}$ would result in less than a $1^{\circ}$ change in the phase of Indian Ocean satellite $B_{z}$ (Fig. 10c), and a 2 percent change (roughly $0.02 \mathrm{nT}$ ) in the amplitude of Northeastern Pacific satellite $B_{z}$ (Fig. 10b) — comparable to errors due to the uncertainty in the tidal source. However, for other regions and magnetic components, changes in the OTEM field caused by different Earth models are much larger than the uncertainty resulting from the tidal current model (see Section 4), suggesting that the OTEM signal is a reliable tool to probe the deep Earth.

\section{SEAFLOOR OBSERVATIONS AND SIMULATIONS}

In this section we estimate the resistivity profile beneath the northeastern Pacific by fitting M2 OTEM observations from the ocean bottom experiment BEMPEX (Luther et al. 1987). Collected on the northeastern Pacific seafloor $\left(41^{\circ} \mathrm{N}, 165^{\circ} \mathrm{W}\right.$, see Fig. 1), the BEMPEX dataset consists of horizontal components at seven electrometers and three-component records at five magnetometers. The data were collected at sample rates of $16-128 \mathrm{hr}^{-1}$ for a year.

The raw time-series were decimated to hourly means and trends in the data were removed. A set of spectral lines, consisting of the principal tides in the long period, diurnal and semi-diurnal bands as well as the solar daily variation and its harmonics, were fit to each time-series in an iterative fashion. The inner loop of the iterative procedure estimates the amplitude and phase of the lines, and the outer loop was used to compute residual variance and assess the significance of each line. In the inner loop, the hourly mean observation can be defined by the sum of sine and cosine terms with nodal corrections (Cartwright \& Tayler 1971). The linear equations of unknown coefficients for each line were solved using a conventional robust approach with Huber weights and QR decomposition. In the outer loop, we computed the multi-taper power spectrum of the residuals after subtracting the estimated lines from the data, and assessed the significance of each line using a likelihood ratio test. The procedure terminates when all $p$-values for significance of a periodic component are below 0.05 . Finally, confidence intervals were placed on the amplitudes and phases using a percentile bootstrap approach. The 0.05 tail probability at each period is apportioned equally between the amplitude and phase. A more detailed description of data processing is provided in the supplementary material.

The observations are fit to a model via a trial-and-error approach, using $0.2^{\circ} \times 0.2^{\circ}$ high-resolution regional forward modelling runs. Fig. 12 shows three representative $1-\mathrm{D}$ resistivity models, among the dozens tried, and Figs 13 and 14 compare predictions and observations. In Fig. 12 the resistivity profile labeled as 'BEMPEX' best reproduces the observations among those tried. The other two models shown are from previously published work: the NoMelt model (red line) is the averaged 1-D model from the 2-D MT survey in the central Pacific $\left(9^{\circ} \mathrm{N}, 145^{\circ} \mathrm{W}\right.$; Sarafian et al. 2015), and the Pac $\mathrm{C}$ model (green line) is the 1-D northwestern Pacific MT model $\left(27^{\circ} \mathrm{N}, 147^{\circ} \mathrm{E}\right.$; Baba et al. 2010). The average lithosphere age in the BEMPEX, NoMelt and Pac $\mathrm{C}$ regions are 78, 70 and $147 \mathrm{Ma}$, respectively. In the BEMPEX experiment, the magnetic field was measured in geomagnetic coordinates, and the magnetic component $H$ in Fig. 14 is the modelled magnetic field rotated to the local geomagnetic north. Error bars in Figs 13 and 14 indicate double-sided 95 per cent confidence intervals, derived as noted above.

In the study region, the amplitudes of the OTEM observations are larger than the global average: the north electric field $E_{x}$ is about $0.35 \mathrm{mV} \mathrm{km}^{-1}$ and the magnetic component $H$ is up to 4 nT. With small error bars, the observed amplitude of $E_{x}$ varies between sites, but the trend can be reproduced by all of the three resistivity models (Fig. 13). Note that the tidal electric field on the seafloor is subject to galvanic distortion (Chave et al. 2004). 


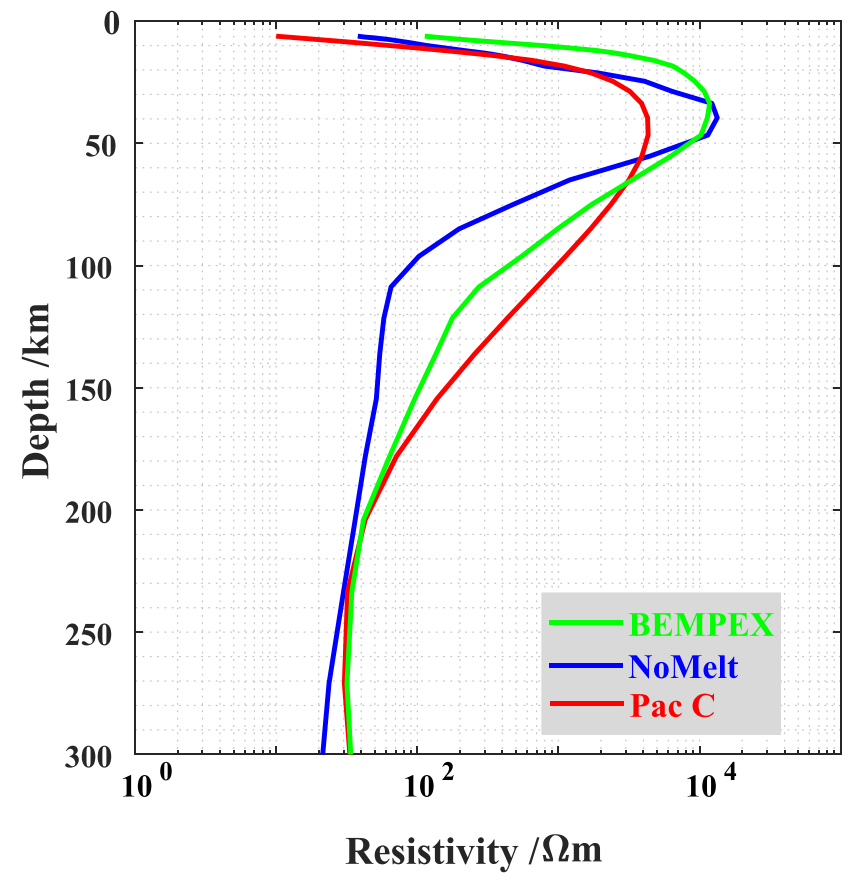

Figure 12. Resistivity profiles of regional models. The green line shows the synthetic model which fits the BEMPEX observation. The blue line shows the averaged 1-D NoMelt MT model in the central Pacific (Sarafian et al. 2015). The red line is 1-D northwestern MT model near the Bonin Trench (Baba et al. 2010).

The prediction of the BEMPEX model fits the amplitude slightly better. However, all predictions fail to fit the observed phase which is scattered. As discussed by Chave (1983), the amplitude of the seafloor horizontal electric field is governed primarily by the electric source current at the measurement point, and so is less dependent on deep resistivity structure, and more sensitive to the conductivityweighted tidal volume transport.

In contrast to the electric field, only the BEMPEX model can both fit the observed amplitude and phase of $H$ (Fig. 14). The predicted phases of the Pac $\mathrm{C}$ model fit the data well, but the amplitudes are $0.5 \mathrm{nT}$ larger than the observations. The predicted amplitudes of the NoMelt model are also $0.5 \mathrm{nT}$ larger and the phases are off by roughly four degrees. According to the sensitivity analysis of OTEM in the northeastern Pacific (see Section 5), the transverse resistance $\rho_{l} H_{l}$ influences the amplitude of seafloor horizontal magnetic field the most. The lithosphere of the NoMelt and Pac C models is either too thin or too conductive; resistance of the model should be increased to achieve a better fit. We also showed in Section 5 that in the study region the phase of the seafloor magnetic field would decrease with a larger ratio of lithospheric and asthenospheric resistivity $\rho_{l} / \rho_{a}$. The NoMelt model fails to match the observed phase due to the dramatic decrease in resistivity at the LAB. The BEMPEX and Pac C models fit the phase better because the resistivity contrast between the lithosphere and asthenosphere is smaller. We also compared the observed and modelled vertical magnetic signal $Z$ at four stations (Fig. S7), the limited data quantity and relatively small amplitude of the $Z$ observations make it difficult to obtain a reasonable degree of fit.

The OTEM observations suggest that in the study area, the transverse resistance from surface to $125 \mathrm{~km}$ depth is about $7.0 \times$ $10^{8} \Omega \mathrm{m}^{2}$, larger than the corresponding resistance in the NoMelt $\left(3.6 \times 10^{8} \Omega \mathrm{m}^{2}\right)$ and Pac $\mathrm{C}\left(2.5 \times 10^{8} \Omega \mathrm{m}^{2}\right)$ models. The resistivity structure of the asthenosphere in the study area is more similar to a Pac C-like model, with a deeper LAB and a more gradual drop in resistivity to a less conductive asthenosphere.

As mentioned above, the NoMelt and Pac C models are from ocean bottom MT surveys which took place on 70 and $147 \mathrm{Myr}$ old oceanic lithosphere, respectively (Müller et al. 2008), and the lithospheric age in the study area is $78 \mathrm{Myr}$. In terms of tectonics, the BEMPEX array was located near the west end of the Mendocino fracture zone in the northeastern Pacific, the NoMelt array was located between the Clarion and Clipperton fracture zones in the central Pacific, and the Pac $\mathrm{C}$ array was located near the Izu-Bonin Trench in the western Pacific. Despite the similar lithospheric age and surface tectonics, the BEMPEX model (78 Myr) does not resemble the NoMelt model, but is closer to an ancient plate without a sharp resistivity drop in the asthenosphere, like the Pac $\mathrm{C}$ model. Baba et al. (2017) argued that a plate cooling model does not predict the thermal structure of the northwestern Pacific, since significant differences were observed in the thickness of the resistive layer with similar age. We also doubt that an age difference of $8 \mathrm{Myr}$ between BEMPEX and NoMelt areas is fully responsible for the observed differences in electrical properties of the lithosphere and underlying asthenosphere. Possibly the difference results from differences in geodynamic setting. For example, the impact of the nearby fractures zones should be further explored.

\section{CONCLUSIONS}

We have developed a new 3-D FD simulation tool for global and regional modelling of the OTEM field. The numerical tool is flexible and can easily be integrated into a commonly used EM inversion modular system (Kelbert et al. 2014), which will allow more extensive (ultimately 3-D) inversion of the OTEM fields observed both with satellite and in situ instruments, and other natural source EM signals, for example, MT and GDS. The global 3-D forward model is used to assess errors in OTEM numerical predictions caused by the uncertainty in tidal models. Our analysis demonstrates that errors are negligible in most of the ocean interior, but may be significant in some coastal areas.

One feature of this work is the 1-D theoretical analysis of the OTEM field, including its modal expansion, relation with ocean tidal currents, and sensitivity to Earth's structure. The results demonstrate that the PM mode of OTEM (the only component present in satellite data) is caused by horizontally divergent flow, and the TM mode (which can be observed horizontal seafloor magnetic fields) is induced by solenoidal tidal currents. It is also shown by the analysis that the PM mode is most sensitive to asthenospheric resistivity and depth, while the TM mode is very sensitive to lithospheric transverse resistance. The 1-D theory can explain many first-order features of the OTEM field in/above the ocean interior far from the coastline, and consequently provides a basic insight into the physical nature of the signal. Nevertheless, some 1-D analytic results are contradicted by the more realistic 3-D numerical modelling results, especially near coastlines, demonstrating the limitations of a simplified 1-D theory. 3-D modelling with full consideration of bathymetry and current source is essential to a quantitative interpretation of OTEM observations.

We have also carefully studied sensitivity of the OTEM field to Earth's structure, demonstrating its relationship to the measurement modality (i.e. satellite or ocean bottom), the data type (amplitude 
(a)

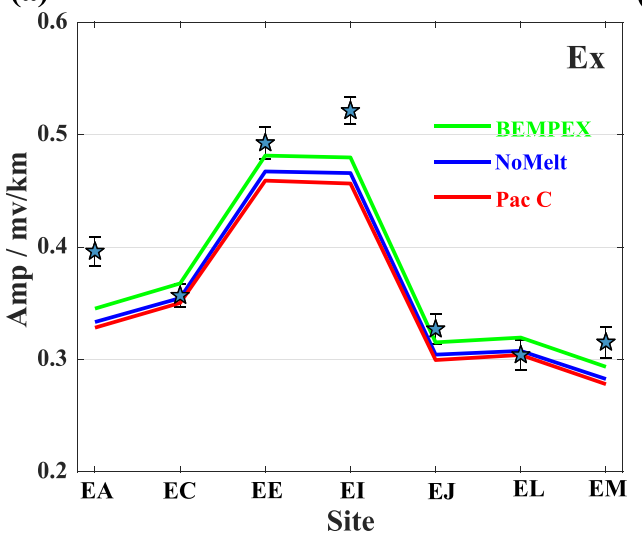

(b)

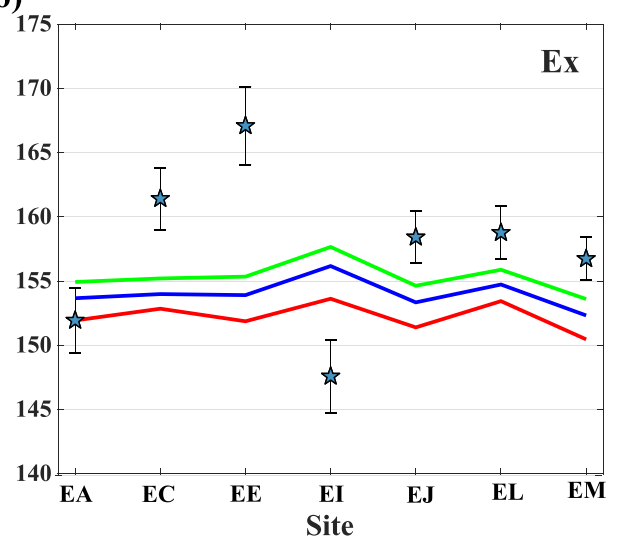

Figure 13. The observed versus predicted electric signal at BEMPEX seafloor stations. (a) The amplitude of $E_{x}$. (b) The phase of $E_{x}$. The light blue pentagrams are observations and their error bars indicate double-sided 95 per cent confidence intervals. Colour legends are similar to Fig. 12.

(a)

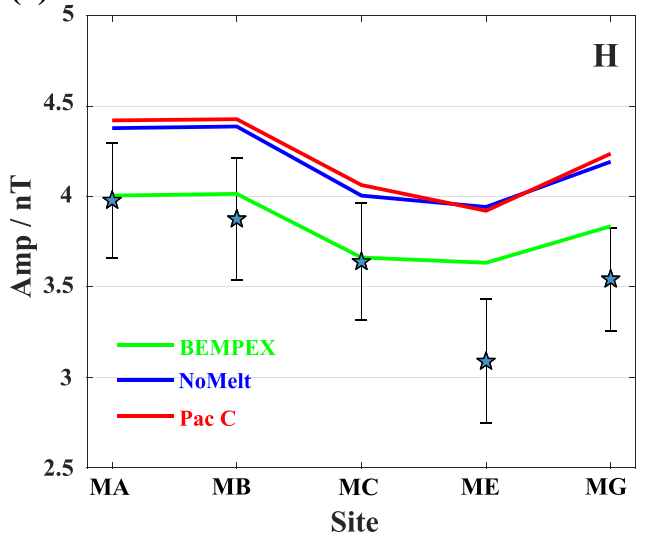

(b)

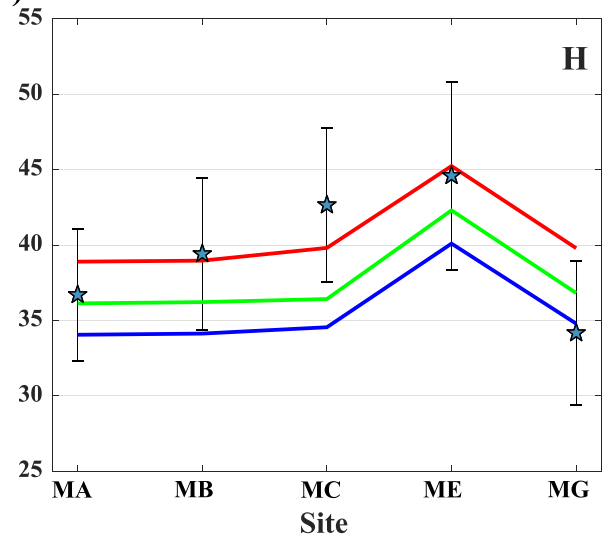

Figure 14. The observed versus predicted magnetic signal at BEMPEX seafloor stations. (a) The amplitude of $H$. (b) The phase of $H$. Symbols are same with Fig. 13.

or phase) as well as tidal flow type (divergent or solenoidal). Exemplified by northeastern Pacific and Indian Ocean regions, satellite data is mostly influenced by the asthenosphere, while the horizontal seafloor magnetic field amplitude is very sensitive to the lithosphere. Phase of horizontal magnetic field on the seafloor is also related to the asthenospheric resistivity. Both the 1-D theoretical analysis and 3-D numerical modelling have proven that satellite and seafloor OTEM observations have different sensitivity to the resistivity model, which clearly indicates that the usage of combined dataset from multiple observation techniques would best constrain the structure of the deep Earth.

This paper is closed with the interpretation of seafloor observations in the northeastern Pacific $\left(41^{\circ} \mathrm{N}, 165^{\circ} \mathrm{W}\right)$. Even though the layered model shows that the lithosphere in the study area is either very resistive or thick (with a transverse resistance of $7.0 \times$ $10^{8} \Omega \mathrm{m}^{2}$ ), and does not favour a very sharp drop in resistivity between the lithosphere and asthenosphere, it is difficult to derive a higher resolution resistivity-depth profile with a single period. In order to produce a less biased resistivity model, a more detailed study of this region calls for the utilization of multiple observations. We plan to address related questions including 3-D resistivity distribution and anisotropy by the joint inversion of the seafloor and satellite OTEM data with more tidal constituents and marine MT data. Hopefully, this can provide new insight into the evolution of oceanic lithosphere and the underlying asthenosphere.

\section{ACKNOWLEDGEMENTS}

This work has been supported by National Natural Science Foundation of China grants 41804072 and 41574104 , and NSF grant EAR1447109. Special thanks to Dr Benjamin Murphy who provided the conductivity-depth profile for 1-D earth model, Dr Min Ding who provided valuable discussion about the oceanic lithosphere and Dr Jeffery Love who provided comments on the stylistics of the manuscript.

\section{REFERENCES}

Baba, K., Chave, A.D., Evans, R.L., Hirth, G. \& Mackie, R.L., 2006. Mantle dynamics beneath the East Pacific Rise at $17^{\circ} \mathrm{S}$ : Insights from the Mantle Electromagnetic and Tomography (MELT) experiment, J. geophys. Res.: Solid Earth, 111, B02101.

Baba, K., Utada, H., Goto, T., Kasaya, T., Shimizu, H. \& Tada, N., 2010. Electrical conductivity imaging of the Philippine Sea upper mantle using seafloor magnetotelluric data, Phys. Earth planet. Inter., 183(1-2), 44-62. 
Baba, K. et al. 2017. Electrical conductivity of old oceanic mantle in the northwestern Pacific I: 1-D profiles suggesting differences in thermal structure not predictable from a plate cooling model, Earth, Planets Space, 69, 111

Backus, G., 1986. Poloidal and toroidal fields in geomagnetic field modeling, Rev. Geophys., 24(1), 75-109.

Cartwright, D. \& Tayler, R., 1971. New computations of the tide-generating potential, Geophys. J. Int., 23(1), 45-73.

Chave, A.D., 1983. On the theory of electromagnetic induction in the Earth by ocean currents, J. geophys. Res., 88(B4), 3531-3542.

Chave, A.D., 1984. The Fréchet derivatives of electromagnetic induction, $J$. geophys. Res.: Solid Earth, 89(B5), 3373-3380.

Chave, A.D. \& Luther, D.S., 1990. Low-frequency, motionally induced electromagnetic fields in the ocean: 1. theory, J. geophys. Res. Oceans, 95(C5), 7185-7200.

Chave, A.D. \& Weidelt, P., 2012. The theoretical basis for electromagnetic induction, in The Magnetotelluric Method: Theory and Practice, pp. 1949, eds Chave, A.D. \& Jones, A.G., Cambridge University Press.

Chave, A.D., Luther, D.S. \& Meinen, C.S., 2004. Correction of motional electric field measurements for galvanic distortion, J. Atmos. Oceanic Technol., 21(2), 317-330.

Dai, L. \& Karato, S.I., 2014. High and highly anisotropic electrical conductivity of the asthenosphere due to hydrogen diffusion in olivine, Earth planet. Sci. Lett., 408, 79-86.

Dushaw, B.D., Egbert, G.D., Worcester, P.F., Cornuelle, B.D., Howe, B.M. \& Metzger, K., 1997. A TOPEX/POSEIDON global tidal model (TPXO. 2 ) and barotropic tidal currents determined from long-range acoustic transmissions, Prog. Oceanogr., 40(1-4), 337-367.

Egbert, G.D. \& Erofeeva, S.Y., 2002. Efficient inverse modeling of barotropic ocean tides, J. Atmos. Oceanic Technol., 19(2), 183-204.

Egbert, G.D. \& Kelbert, A., 2012. Computational recipes for electromagnetic inverse problems, Geophys. J. Int., 189(1), 251-267.

Egbert, G.D., Bennett, A.F. \& Foreman, M.G., 1994. TOPEX/POSEIDON tides estimated using a global inverse model, J. geophys. Res. Oceans, 99(C12), 24821-24852.

Evans, R.L. et al., 1999. Asymmetric electrical structure in the mantle beneath the east Pacific rise at $17^{\circ} \mathrm{S}$, Science, 286(5440), 752-756.

Evans, R.L., Chave, A.D. \& Booker, J.R., 2002. On the importance of offshore data for magnetotelluric studies of ocean-continent subduction systems, Geophys. Res. Lett., 29(9), 16-1.

Filloux, J.H., 1977. Ocean-floor magnetotelluric sounding over north central pacific, Nature, 269(5626), 297.

Grayver, A.V. \& Olsen, N., 2019. The magnetic signatures of the M2, N2, and $\mathrm{O} 1$ oceanic tides observed in Swarm and CHAMP satellite magnetic data, Geophys. Res. Lett., 46(8), 4230-4238.

Grayver, A.V., Schnepf, N.R., Kuvshinov, A.V., Sabaka, T.J., Manoj, C. \& Olsen, N., 2016. Satellite tidal magnetic signals constrain oceanic lithosphere-asthenosphere boundary, Sci. Adv., 2(9), e1600798.

Grayver, A.V., Munch, F.D., Kuvshinov, A.V., Khan, A., Sabaka, T.J. \& Tøffner-Clausen, L., 2017. Joint inversion of satellite-detected tidal and magnetospheric signals constrains electrical conductivity and water content of the upper mantle and transition zone, Geophys. Res. Lett., 44(12), 6074-6081.

Irrgang, C., Saynisch, J. \& Thomas, M., 2019. Estimating global ocean heat content from tidal magnetic satellite observations, Scient. Rep., 9(1), 7893.

Junge, A., 1988. The telluric field in northern Germany induced by tidal motion in the North Sea, Geophys. J. Int., 95(3), 523-533.

Kelbert, A., Schultz, A. \& Egbert, G., 2009. Global electromagnetic induction constraints on transition-zone water content variations, Nature, 460(7258), 1003-1006.

Kelbert, A., Meqbel, N., Egbert, G.D. \& Tandon, K., 2014. ModEM: a modular system for inversion of electromagnetic geophysical data, Comput. Geosci., 66, 40-53.

Key, K. \& Constable, S., 2011. Coast effect distortion of marine magnetotelluric data: insights from a pilot study offshore northeastern japan, Phys. Earth planet. Inter., 184(3-4), 194-207.
Key, K., Constable, S., Liu, L. \& Pommier, A., 2013. Electrical image of passive mantle upwelling beneath the northern East Pacific Rise, Nature, 495(7442), 499-502.

Kuvshinov, A., 2008. 3-D global induction in the oceans and solid earth: recent progress in modeling magnetic and electric fields from sources of magnetospheric, ionospheric and oceanic origin, Surv. Geophys., 29(2), 139-186.

Kuvshinov, A. \& Olsen, N., 2006. A global model of mantle conductivity derived from 5 years of CHAMP, ørsted, and SAC-C magnetic data, Geophys. Res. Lett., 33(18), L18301.

Kuvshinov, A., Junge, A. \& Utada, H., 2006. 3-D modelling the electric field due to ocean tidal flow and comparison with observations, Geophys. Res. Lett., 33(6), L06314.

Larsen, J., 1968. Electric and magnetic fields induced by deep sea tides, Geophys. J. Int., 16(1), 47-70.

Le Provost, C., 2001. Ocean tides, in Satellite Altimetry and Earth Sciences, pp. 267-303, eds Fu, L.-L. \& Cazenave, A., Academic Press.

Luther, D.S., Chave, A.D. \& Filloux, J.H., 1987. BEMPEX: A study of barotropic ocean currents and lithospheric electrical conductivity, EOS, Trans. Am. Geophys. Un., 68(27), 618-629.

Luther, D.S., Filloux, J.H. \& Chave, A.D., 1991. Low-frequency, motionally induced electromagnetic fields in the ocean: 2. Electric field and Eulerian current comparison, J. geophys. Res. Oceans, 96(C7), 12797-12814.

Mackie, R.L., Smith, J.T. \& Madden, T.R., 1994. Three-dimensional electromagnetic modeling using finite difference equations: The magnetotelluric example, Radio Sci., 29(4), 923-935.

Matsuno, T. et al., 2010. Upper mantle electrical resistivity structure beneath the central mariana subduction system, Geochem. Geophys. Geosyst., 11(9), Q09003.

Maus, S. \& Kuvshinov, A., 2004. Ocean tidal signals in observatory and satellite magnetic measurements, Geophys. Res. Lett., 31(15), L15313.

Minami, T., 2017. Motional induction by tsunamis and ocean tides: 10 years of progress, Surv. Geophys., 38(5), 1097-1132.

Müller, R.D., Sdrolias, M., Gaina, C. \& Roest, W.R., 2008. Age, spreading rates, and spreading asymmetry of the world's ocean crust, Geochem. Geophys. Geosyst., 9(4), Q04006.

Munch, F.D., Grayver, A.V., Kuvshinov, A. \& Khan, A., 2018. Stochastic inversion of geomagnetic observatory data including rigorous treatment of the ocean induction effect with implications for transition zone water content and thermal structure, J. geophys. Res.: Solid Earth, 123(1), 3151.

Naif, S., Key, K., Constable, S. \& Evans, R., 2013. Melt-rich channel observed at the lithosphere-asthenosphere boundary, Nature, 495(7441), 356.

Parkinson, W. \& Jones, F., 1979. The geomagnetic coast effect, Rev. Geophys., 17(8), 1999-2015.

Püthe, C., Kuvshinov, A., Khan, A. \& Olsen, N., 2015. A new model of Earth's radial conductivity structure derived from over $10 \mathrm{yr}$ of satellite and observatory magnetic data, Geophys. J. Int., 203(3), 1864-1872.

Ray, R.D. \& Egbert, G.D., 2017. Tides and satellite altimetry, in Satellite Altimetry over Oceans and Land Surfaces, pp. 427-458, eds Stammer, D. \& Cazenave, A., CRC Press.

Ray, R.D., Eanes, R.J., Egbert, G.D. \& Pavlis, N.K., 2001. Error spectrum for the global $\mathrm{M}_{2}$ ocean tide, Geophys. Res. Lett., 28(1), 21-24.

Sabaka, T.J., Olsen, N., Tyler, R.H. \& Kuvshinov, A., 2015. CM5, a preSwarm comprehensive geomagnetic field model derived from over $12 \mathrm{yr}$ of CHAMP, ørsted, SAC-C and observatory data, Geophys. J. Int., 200(3), $1596-1626$.

Sabaka, T.J., Tyler, R.H. \& Olsen, N., 2016. Extracting ocean-generated tidal magnetic signals from Swarm data through satellite gradiometry, Geophys. Res. Lett., 43(7), 3237-3245.

Sanford, T.B., 1971. Motionally induced electric and magnetic fields in the sea, J. geophys. Res., 76(15), 3476-3492.

Sarafian, E., Evans, R.L., Collins, J.A., Elsenbeck, J., Gaetani, G.A., Gaherty, J.B., Hirth, G. \& Lizarralde, D., 2015. The electrical structure of the central Pacific upper mantle constrained by the NoMelt experiment, Geochem. Geophys. Geosyst., 16(4), 1115-1132. 
Saynisch, J., Petereit, J., Irrgang, C. \& Thomas, M., 2017. Impact of oceanic warming on electromagnetic oceanic tidal signals: A CMIP5 climate model-based sensitivity study, Geophys. Res. Lett., 44(10), 4994-5000.

Schnepf, N.R., Manoj, C., Kuvshinov, A., Toh, H. \& Maus, S., 2014. Tidal signals in ocean-bottom magnetic measurements of the northwestern Pacific: observation versus prediction, Geophys. J. Int., 198(2), $1096-1110$.

Schnepf, N.R., Kuvshinov, A. \& Sabaka, T., 2015. Can we probe the conductivity of the lithosphere and upper mantle using satellite tidal magnetic signals?, Geophys. Res. Lett., 42(9), 3233-3239.

Schnepf, N.R., Nair, M., Maute, A., Pedatella, N.M., Kuvshinov, A. \& Richmond, A.D., 2018. A comparison of model-based ionospheric and ocean tidal magnetic signals with observatory data, Geophys. Res. Lett., 45(15), 7257-7267.

Stammer, D. et al., 2014. Accuracy assessment of global barotropic ocean tide models, Rev. Geophys., 52(3), 243-282.

Taguchi, E., Stammer, D. \& Zahel, W., 2014. Inferring deep ocean tidal energy dissipation from the global high-resolution data-assimilative hamtide model, J. geophys. Res. Oceans, 119(7), 4573-4592.

Thébault, E. et al., 2015. International geomagnetic reference field: the 12th generation, Earth, Planets Space, 67(1), 79.

Tyler, R.H., Maus, S. \& Lühr, H., 2003. Satellite observations of magnetic fields due to ocean tidal flow, Science, 299(5604), 239-241.

Tyler, R.H., Boyer, T.P., Minami, T., Zweng, M.M. \& Reagan, J.R., 2017. Electrical conductivity of the global ocean, Earth, Planets Space, 69(1), 156.

Uyeshima, M. \& Schultz, A., 2000. Geoelectromagnetic induction in a heterogeneous sphere: a new three-dimensional forward solver using a conservative staggered-grid finite difference method, Geophys. J. Int., 140(3), 636-650.

Velímskỳ, J., Martinec, Z. \& Everett, M.E., 2006. Electrical conductivity in the earth's mantle inferred from CHAMP satellite measurementsI. data processing and 1-D inversion, Geophys. J. Int., 166(2), 529-542.

Velímský, J., Grayver, A., Kuvshinov, A. \& Šachl, L., 2018. On the modelling of M2 tidal magnetic signatures: effects of physical approximations and numerical resolution, Earth, Planet Space, 70(1), 192.

Wannamaker, P.E., Booker, J.R., Jones, A.G., Chave, A.D., Filloux, J.H., Waff, H.S. \& Law, L.K., 1989. Resistivity cross section through the Juan de Fuca subduction system and its tectonic implications, J. geophys. Res.: Solid Earth, 94(B10), 14127-14144.

Whittaker, J.M., Goncharov, A., Williams, S.E., Müller, R.D. \& Leitchenkov, G., 2013. Global sediment thickness data set updated for the australianantarctic southern ocean, Geochem. Geophys. Geosyst., 14(8), 32973305 .

\section{SUPPORTING INFORMATION}

Supplementary data are available at $G J I$ online.

Figure S1 The sea surface height variation and phase of $M 2$ tidal constituent predicted by TPXO8 (Egbert \& Erofeeva 2002). The cotidal lines (white lines) are spaced at phase interval of $60^{\circ}$.

Figure S2 Contour plot of phase of PM (left-hand column) and TM (right-hand column) response function varying with (a)-(b) lithospheric resistivity $\rho_{l}$ and the LAB depth. The solid and dotted contours are for models in which $\rho_{a}$ is 50 and $200 \Omega \mathrm{m}$, respectively. The black dashed line in $\mathrm{b}$ denotes the line $\rho_{l} H_{l}=$ const. (c)-(d) asthenospheric resistivity $\rho_{a}$ and the LAB depth with fixed lithospheric resistance $\rho_{l} H_{l}$. The solid and dotted contours are for models in which the resistance is $8 \times 10^{8}$ and $4 \times 10^{8} \Omega \mathrm{m}^{2}$, respectively.

Figure S3 The amplitude of satellite $B_{z}$ component in geomagnetic model CM5 (Sabaka et al. 2015).

Figure S4 The phase of the M2 OTEM field. (a) The vertical magnetic component $B_{z}$ at satellite altitude $(430 \mathrm{~km})$ (b) The north magnetic component $B_{x}$ on the seafloor.

Figure S5 Changes in the phase of magnetic signal when the LAB depth increases from 70 to $130 \mathrm{~km}$ (the left-hand column), and the mantle water content increases from 100 to $200 \mathrm{ppm}$ (the right-hand column). (a)-(b) The vertical magnetic signal Bz at satellite altitude. (c)-(d) The vertical magnetic signal $B_{z}$ on the Earth's surface. (e)(f) The north component $B_{x}$ on the seafloor. Note that the scattered outliers are located in regions where the amplitude and phase are small, so a fluctuation in the real or imaginary part of magnetic field can result in large phase shift, but the modelling results are still reliable.

Figure S6 The pseudocolour plot of seafloor $B_{z}$ component in the Indian Ocean (left-hand column) and northeastern Pacific (righthand column) as a function of $\rho_{a}$ and $\rho_{l} H_{l}$. (a)-(b) The relative difference in amplitude. (c)-(d) The absolute differences in phase. Other symbols are similar to Fig. 9 in the main text.

Figure S7 The observed versus predicted magnetic signal at BEMPEX seafloor stations. (a) The amplitude of $Z$. (b) The phase of $Z$. Symbols are same with Fig. 13.

Please note: Oxford University Press is not responsible for the content or functionality of any supporting materials supplied by the authors. Any queries (other than missing material) should be directed to the corresponding author for the paper. 